Swarthmore College

Works

6-8-2001

\title{
Computational Study Of Molecular Hydrogen In Zeolite Na-A. II. Density Of Rotational States And Inelastic Neutron Scattering Spectra
}

Jennifer A. MacKinnon , '95

J. Eckert

D. F. Coker

Amy Lisa Graves

Swarthmore College, abug1@swarthmore.edu

Follow this and additional works at: https://works.swarthmore.edu/fac-physics

Part of the Physics Commons

Let us know how access to these works benefits you

\section{Recommended Citation}

Jennifer A. MacKinnon , '95; J. Eckert; D. F. Coker; and Amy Lisa Graves. (2001). "Computational Study Of Molecular Hydrogen In Zeolite Na-A. II. Density Of Rotational States And Inelastic Neutron Scattering Spectra". Journal Of Chemical Physics. Volume 114, Issue 22. 10137-10150. DOI: 10.1063/1.1352733 https://works.swarthmore.edu/fac-physics/177

This work is brought to you for free by Swarthmore College Libraries' Works. It has been accepted for inclusion in Physics \& Astronomy Faculty Works by an authorized administrator of Works. For more information, please contact myworks@swarthmore.edu. 


\section{AD| $\begin{aligned} & \text { The Journal of } \\ & \text { Chemical Physics }\end{aligned}$}

Computational study of molecular hydrogen in zeolite $\mathrm{Na}-\mathrm{A}$. II. Density of rotational states and inelastic neutron scattering spectra

Jennifer A. MacKinnon, Juergen Eckert, David F. Coker, and Amy L. R. Bug

Citation: The Journal of Chemical Physics 114, 10137 (2001); doi: 10.1063/1.1352733

View online: http://dx.doi.org/10.1063/1.1352733

View Table of Contents: http://scitation.aip.org/content/aip/journal/jcp/114/22?ver=pdfcov

Published by the AIP Publishing

\section{Articles you may be interested in}

Probing the hydrogen equilibrium and kinetics in zeolite imidazolate frameworks via molecular dynamics and quasi-elastic neutron scattering experiments

J. Chem. Phys. 138, 034706 (2013); 10.1063/1.4774375

Hydrogen adsorption in the NaA zeolite: A comparison between numerical simulations and experiments

J. Chem. Phys. 112, 5991 (2000); 10.1063/1.481201

Computational study of molecular hydrogen in zeolite Na-A. I. Potential energy surfaces and thermodynamic separation factors for ortho and para hydrogen

J. Chem. Phys. 111, 7599 (1999); 10.1063/1.480104

Ro-vibrational Stark effect on H 2 and D 2 molecules adsorbed in NaA zeolite

J. Chem. Phys. 110, 5943 (1999); 10.1063/1.478494

Adsorption and coadsorption of molecular hydrogen isotopes in zeolites. II. Infrared analyses of H $2, H D$, and D

2 in $\mathrm{NaA}$

J. Chem. Phys. 109, 6469 (1998); 10.1063/1.477292

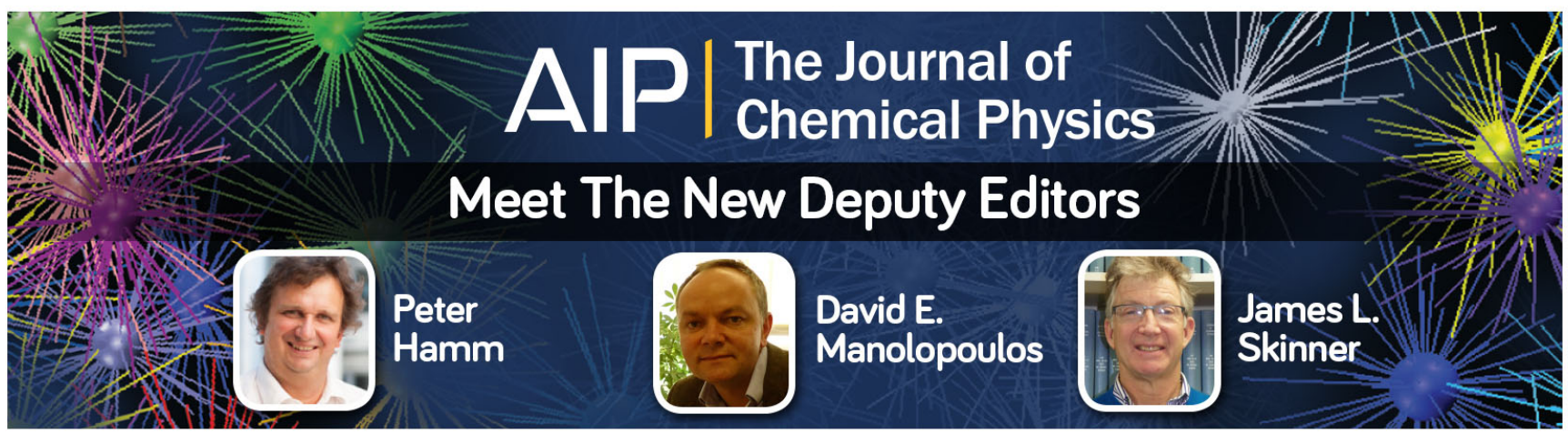




\title{
Computational study of molecular hydrogen in zeolite $\mathrm{Na}-\mathrm{A}$. II. Density of rotational states and inelastic neutron scattering spectra
}

\author{
Jennifer A. MacKinnon ${ }^{\text {a) }}$ \\ Department of Physics and Astronomy, Swarthmore College, Swarthmore, Pennsylvania 19081 \\ Juergen Eckert \\ Los Alamos National Laboratory, Los Alamos, New Mexico 87545 \\ David F. Coker \\ Department of Chemistry, Boston University, Boston, Massachusetts 02215
}

Amy L. R. Bug ${ }^{\text {b) }}$

Department of Physics and Astronomy, Swarthmore College, Swarthmore, Pennsylvania 19081

(Received 20 June 2000; accepted 12 January 2001)

\begin{abstract}
Part I of this series [J. Chem. Phys. 111, 7599 (1999)] describes a simulation of $\mathrm{H}_{2}$ adsorbed within zeolite $\mathrm{Na}-\mathrm{A}$ in which a block Lanczos procedure is used to generate the first several (9) rotational eigenstates of $\mathrm{H}_{2}$, modeled as a rigid rotor, and equilibrated at a given temperature via Monte Carlo sampling. Here, we show that rotational states are strongly perturbed by the electrostatic fields in the solid. Wave functions and densities of rotational energy states are presented. Simulated neutron spectra are compared with inelastic neutron scattering data. Comparisons are made with IR spectra in which rotational levels may appear due to rovibrational coupling. (C) 2001 American Institute of Physics. [DOI: 10.1063/1.1352733]
\end{abstract}

\section{INTRODUCTION}

In part I of this series, ${ }^{1}$ we introduced a Monte Carlo (MC) simulation of adsorbed $\mathrm{H}_{2}$ in the zeolite $\mathrm{Na}-\mathrm{A}$. Realistic guest-host interaction potentials and a quantum mechanical treatment of the rotations of the $\mathrm{H}_{2}$ molecule were used within the context of a MC sampling procedure. We discussed generic types of potential energy surface encountered by the hindered $\mathrm{H}_{2}$ rotor within the solid, and the effect that these surfaces have on the "separation factor," $S$ $\equiv\left(Q_{o} / Q_{p}\right)_{\text {adsorbed }} /\left(Q_{o} / Q_{p}\right)_{\text {free }}$, where $Q_{o, p}$ are the partition functions of ortho-and para- $\mathrm{H}_{2}$, respectively. $S$ is a measure of the preferential adsorbtion of ortho- over para- $\mathrm{H}_{2}$ by the solid.

A few of the main conclusions of part I are (i) $\mathrm{H}_{2}$ orientation is typically "side-on" to the closest cation because of the predominance of quadrupole interactions, ${ }^{2-8}$ and these interactions tend to be important in determining the shape of the rotational potential energy surface, and the separation factor. (ii) At $300 \mathrm{~K}$ the hydrogen is free to explore the extent of the supercage, and is also free to cross through the octagonal doorways between cages. At $100 \mathrm{~K}$ such crossings are less common during simulation, and hydrogen shows a preference for the vicinity of both a $\mathrm{Na}_{\text {II }}$ and $\mathrm{Na}_{\text {III }}$ ion. At 50 $\mathrm{K}$ and below, the hydrogen settles into a potential well in this vicinity. This is consistent with a picture formulated in a great number of spectroscopic studies, ${ }^{6,9-12}$ that at low temperature hydrogen is adsorbed "specifically" at certain sites. For example, quasielastic neutron scattering studies have de-

\footnotetext{
${ }^{a)}$ Current address: Applied Physics Lab, University of Washington, Seattle, WA 98195 .

${ }^{b)}$ Author to whom correspondence should be addressed.
}

termined that virtually all adsorbed $\mathrm{H}_{2}$ molecules are immobile on the timescale of the experiment (tens of ps) at $50 \mathrm{~K}$, while none are similarly immobile at $150 \mathrm{~K} .{ }^{13}$ (iii) Two adsorption sites in our model were termed the " $A$ " and " $B$ " sites, on either side of a $\mathrm{Na}_{\text {III }}$ cation. Figure 6 of part I depicts these locations. Thus the model contains more than one type of site which compete to bind $\mathrm{H}_{2}$ at low temperature. But rather than a site corresponding to a single type of cation (e.g., type I, II, or III) as some authors have posited, it is more accurate to think of these sites as a region whose adsorptivity comes from the presence of a number of nearby cations and anions. (iv) The potential in which hydrogen moves can be thought of as one with both an out-of-plane and in-plane barrier to rotation. The heights of these two barriers, conventionally referred to as $\lambda$ and $2 \mu$, vary with location in the zeolite, and are typically found to be several rotational constants, $B$, in magnitude.

In the current paper, we will discuss the rotational behavior of $\mathrm{H}_{2}$ within zeolite $\mathrm{Na}-\mathrm{A}$, examining the rotational wave functions and the densities of states. We will present direct measurements of the ortho- to para- $\mathrm{H}_{2}$ rotational tunneling transition by inelastic neutron scattering (INS). These experimental data will be compared with our computed rotational potential barriers, and with simulated neutron spectra. We will also propose a small modification to the traditional two parameter $(\lambda, \mu)$ model of a hindered rotor; one that better reproduces both the potential surfaces and the eigenspectra seen in our system.

\section{METHODS}

Details of our methods are given in Sec. II of part I. To summarize these briefly: we model the dehydrated zeolite 
$\mathrm{Na}-\mathrm{A} .{ }^{14}$ The crystal is cubic with unit cell diameter $12.3 \AA$, and space group Pm $3 \mathrm{~m}$ if one does not distinguish between $\mathrm{Si}$ and $\mathrm{Al}$; and we do not make this distinction in the model. The potential energy for the $\mathrm{H}_{2}$ guest is a sum of terms:

$$
U=U_{\text {disp }}+U_{\text {rep }}+U_{\text {pol }}+U_{\text {multipole }},
$$

consisting of, in this order in Eq. (1), a (London) dispersion attraction, short-ranged repulsion; linear polarization and quadrupolar interaction with the electric field from $\mathrm{Na}$ and $\mathrm{O}$ ions. Ions of a given species share the same potential parameters (see Table II of part I for a listing). For most of the data generated in the current study, we use partial charges $q_{\mathrm{Na}}$ $=0.60 e$ and $q_{O}=-0.15 e$. (Very recently, a grand canonical Monte Carlo study of high pressure $\mathrm{H}_{2}$ in $\mathrm{Na}-\mathrm{A}$ has employed $\mathrm{Na}$ charges which are quite different for the three crystallographically distinct $\mathrm{Na}$ locations. ${ }^{15}$ ) We assume that the energy of $\mathrm{H}_{2}$ at a location $\mathbf{R}$ in the crystal may be separated into a center-of-mass and an orientational term:

$$
V=V_{0}(\mathbf{R})+V_{\mathbf{R}}(\Omega) .
$$

(Note: In paper I we adopted $\mathbf{r}$ as notation for the center-ofmass of the molecule; the notation here is $\mathbf{R}$.) The first term of Eq. (1), $U_{\text {disp }}$, corresponds to $V_{0}(\mathbf{R})$; the remaining terms constitute $V_{\mathbf{R}}(\Omega)$.

A cubic simulation cell of width $r_{c}=24.6 \AA$ ( 2 unit cells) is used. Periodic boundary conditions are employed, which necessitates cutting off interaction potentials at a range of $r_{c} / 2$ or less. The electrostatic interactions are additionally truncated using a "switching" function, which imposes an exponential dropoff on the electric field beyond an intermediate radius.

We assume that the hydrogenic wave function may be represented as a product of a center-of-mass and an orientational term:

$$
\Psi=\Psi_{0}(\mathbf{R}) \phi_{\mathbf{R}}(\Omega) .
$$

The assumption here is that the angular Schrödinger equation can be solved for each position $\mathbf{R}$; an adiabatic approximation which is supported in Appendix A of part I. A further assumption in our model is that the center-of-mass "motion" of the molecule can be treated classically:

$$
\Psi_{0}(\mathbf{R})=\delta(\mathbf{R}-\mathbf{R}(t)) .
$$

Hence we do not report on the vibrational levels of the molecule as a whole in the zeolitic host. We are left with the task of solving the Schrödinger equation for the rotational motion of hydrogen:

$$
\left(-\frac{\hbar^{2}}{2 I} \hat{L}^{2}+V_{\mathbf{R}}(\Omega)\right) \phi_{\mathbf{R}}^{k}=\epsilon_{\mathbf{R}}^{k} \phi_{\mathbf{R}}^{k},
$$

and this is achieved with a block Lanczos algorithm. ${ }^{16}$ The algorithms yields energy levels and also eigenfunctions, $\phi_{\mathbf{R}}^{k}(\Omega)$, where $\Omega$ is chosen on a discrete $(32 \times 32)$ angular

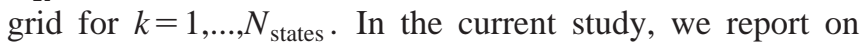
$N_{\text {states }}=9$ rotational eigenstates. The internuclear distance $d_{0}$, in terms of which $I=m d_{0}^{2} / 2$, is taken as $0.77 \AA$.

The center-of-mass performs a random walk through the host. From a location $\mathbf{R}$, a trial step to a new location $\mathbf{R}^{\prime}$ is made. This move is accepted or rejected, in standard Metropolis Monte Carlo fashion, ${ }^{17}$ based on the ratio of partition functions $Q(\mathbf{R}) / Q\left(\mathbf{R}^{\prime}\right)$, where

$$
Q(\mathbf{R})=e^{-\beta V_{0}(\mathbf{R})} \sum_{k=0}^{N_{\text {states }}} g_{k} e^{-\beta \epsilon_{R}^{k}}
$$

with $\beta=1 / k_{B} T$ and $g_{k}$ a degeneracy factor arising from nuclear spin. That is, $g_{k}$ is 1 for the spin singlet state, parahydrogen, and 3 for the triplet, orthohydrogen. ${ }^{18}$ The determination of the nuclear spin of state $k$ is made based on the inversion symmetry of the corresponding wave function calculated with the Lanczos algorithm.

\section{ROTATIONAL ORBITALS, ENERGIES, AND NEUTRON SPECTRA}

\section{A. Adsorbate locations and orientations}

A precise knowledge of the state of an adsorbate molecule in zeolites both facilitates understanding its interaction with the host and determines the functionality of such systems for gas storage, separation, and catalysis. Spectroscopic methods have been useful in revealing details about the state of $\mathrm{H}_{2}$ adsorbed in zeolites. Since the first study demonstrating the IR activation of the fundamental vibration of homonuclear diatomics by the strong electric fields in a zeolite, ${ }^{19}$ there have been numerous IR spectroscopic studies on $\mathrm{H}_{2}{ }^{6,7,9-12,20-28}$ The IR lines identified in these papers have been attributed to intramolecular vibrational motions, centerof-mass (CM) motions, and rotational motions coupled to intramolecular vibrations. Neutron studies ${ }^{29-34}$ have revealed information about translational motions, and importantly, about hindered rotational levels inaccessible to IR because the transitions involve the flipping of a nuclear spin.

One of the challenges is, of course, to distinguish which modes give rise to which spectral bands. One distinguishing tool is the existence of spectroscopic selection rules. Another way to distinguish a rotation from a $\mathrm{CM}$ vibration of $\mathrm{H}_{2}$ is to note whether the band appears both on the energy gain and energy loss sides of the spectrum. Another tool is to substitute $\mathrm{HD}$ or $\mathrm{D}_{2}$ (more difficult in INS because of deuterium's small neutron cross section) and look for an isotope effect. Vibrational energies will be reduced in this case by a factor of $\sqrt{2}$, while the hindered rotational isotope effect is slightly more complicated to calculate (see below). Finally, coadsorption studies may be used to probe for multiple adsorbant sites. $^{31,35}$ Care is required when using these tools, for ambiguity is possible. Consider the INS study of $\mathrm{H}_{2}$ in $\mathrm{Na}-\mathrm{Ca}-\mathrm{A} .{ }^{30}$ A line at approximately $5.6 \mathrm{meV}$ in the $\mathrm{H}_{2}$ spectrum naturally seemed to correspond to a line at $4 \mathrm{meV}$ in the $\mathrm{D}_{2}$ spectrum. While the ratio of these energies is in excellent agreement with the CM vibrational isotope effect, the line was determined not to be a $\mathrm{CM}$ vibration, but rather a rotation, because it was absent on the energy-loss side of the HD spectrum. (The corresponding rotational line in the $\mathrm{D}_{2}$ spectrum would be at $0.9 \mathrm{meV}$, at an energy too low to resolve in this study.) Both IR and INS studies can certainly benefit from detailed, atomistic calculations in order to associate the observed excitation with a particular mode of motion, and adsorption in a particular manner, at a particular 


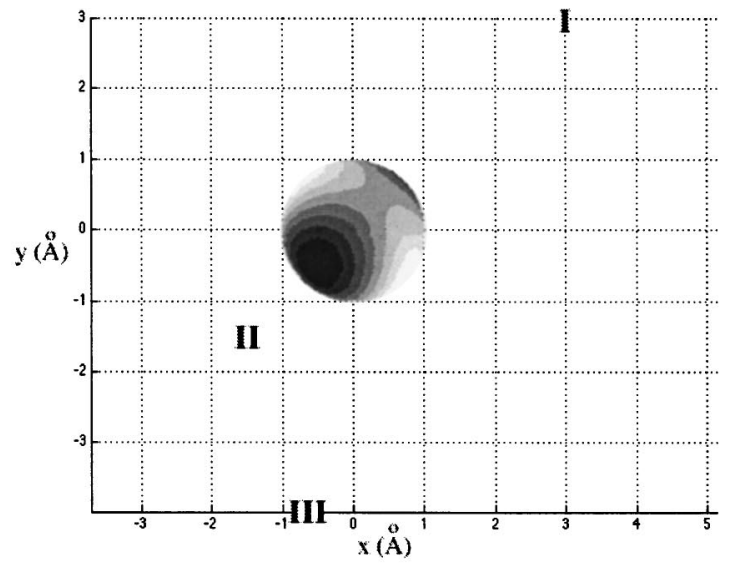

(a)

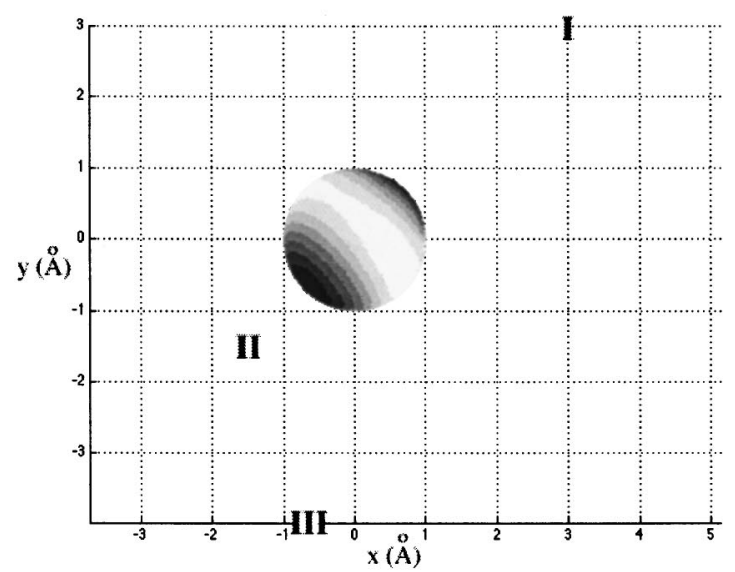

(b)

FIG. 1. (a) Hypothetical potential surface for $10 \mathrm{~K}$ position discussed in text, produced by all $\mathrm{O}$ atoms but only the three closest cations, with positions noted as I, II, and III. (b) Hypothetical potential surface for $10 \mathrm{~K}$ position discussed in text, as produced by only the three closest cations, with positions shown as I, II, and III.

site. Therein lies the importance of studies like Refs. 6, 8, 9, $11,36-39$, which establish this association for IR vibrational shifts. The current study hopes to do the same for neutron rotational scattering spectra. A discussion of how to extend the scope of the analysis to CM modes is presented in Ref. 40.

As was discussed in Sec. III of part I, one might look into the importance of each host atom and each type of term in Eq. (1) in determining the potential, and hence the binding energy, the orientation of the bond axis, and the ease of rotational tunneling of $\mathrm{H}_{2}$ at various locations in the host. In order to try to discern the general principles buried in this wealth of possibilities, we will restrict ourselves to understanding which atoms and types of interaction produce the angular variation in the potential which is relevant to hindering the rotation of $\mathrm{H}_{2}$ at room temperature and below.

Figure 1 is a depiction of a typical adsorption site for $\mathrm{H}_{2}$ at $10 \mathrm{~K}$, with the positions of the three closest zeolite cations indicated schematically. (See Fig. 8 of part I for more details.) The three closest sodium ions to the hydrogen are of type II ( $2.3 \AA$ distant) and types I and III (both approximately $4.6 \AA$ distant). One might compare this proximity to the $\mathrm{Na}_{\text {II }}$ with $\mathrm{HF}$, SCF, and MP2 quantum chemical

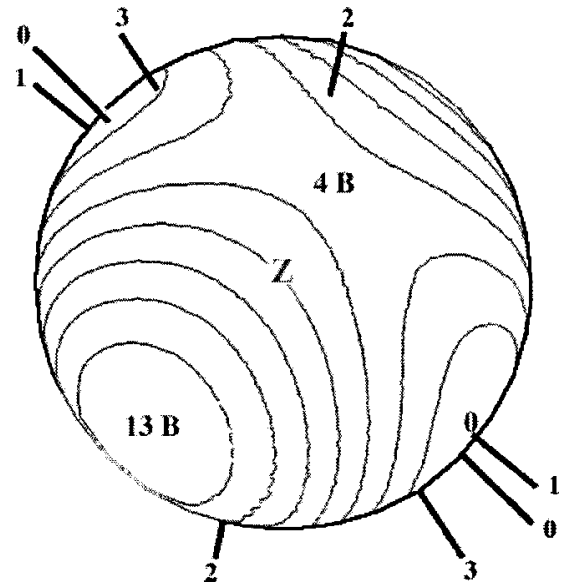

FIG. 2. Potential surface for $10 \mathrm{~K}$ position discussed in text. Saddle point is approximately $4.3 \approx 4 \mathrm{~B}$ above minimum. Average orientations of the hydrogen bond axes, as determined from the calculated angular wave function, are shown for the ground through the third excited states.

calculations ${ }^{3-5,41,42}$ which predict a minimum-energy separation of around $2.5 \AA$ between $\mathrm{H}_{2}$ and a single sodium cation.

Figure 1(a) shows the potential surface that would arise from only the three closest cations, together with all of the oxygen anions in the model. This reproduces the full potential surface, shown in Fig. 2, in semiquantitative detail. The potential energy surface is the generic type [see Fig. 2(c) of part I] with out of- and in-plane hindering. The in-plane barrier (saddle point) retains its approximate angular position when the additional zeolitic cations are allowed to contribute, as in Fig. 2; it is displaced between Figs. 1(a) and 2 by an angle of only $6^{\circ}$. The height of the in-plane barrier on the full surface (Fig. 2) is $350 \mathrm{~K}=4.3 \mathrm{~B}$, while limiting contributions to only the closest three cations [Fig. 1(a)] corresponds to an in-plane barrier height of $500 \mathrm{~K}=6.2 \mathrm{~B}$. (Note that a number of energy units will be used in this paper. For conversion, please note that $1.00 \mathrm{~K}=0.695 \mathrm{~cm}^{-1}$ $=0.00819 \mathrm{~kJ} / \mathrm{mole}=8.62 \mathrm{meV}$.)

In a sense, the presence of the single closest cation (II) is enough to reproduce the qualitative features of the interaction with all cations. So long as oxygens are also retained in the model, cation II alone will produce an in-plane barrier with a height of $5.6 \mathrm{~B}$, and a location only $20^{\circ}$ away from its position when all ions are included in the model. However, if oxygens are removed from the model, the character of the potential surface changes in a meaningful way. To demonstrate this, Fig. 1(b) shows the potential produced by only the three closest cations, but with no contribution from oxygens. The cationic interactions are dominated by the quadrupole term in Eq. (1); no in-plane barrier can exist with cation II alone. When cations III and I (and more) are added, a small in-plane barrier of around $100 \mathrm{~K}=1 \mathrm{~B}$ appears, though it is displaced by more than $60^{\circ}$ from its position when all of the ions (Fig. 2) are present in the model.

In contrast to the situation with sodium cations described above, it seems inappropriate to single out the closest few oxygen anions as contributing most strongly to the qualitative shape of the $\mathrm{O}-\mathrm{H}_{2}$ potential surface. While each oxygen alone would produce a "librator" potential, superposition of 
these (particularly from the nearby 8-ring) produce a band of librational minima, hence a potential which has the character of a "planar rotor," with a depth of $840 \mathrm{~K}=10.2 \mathrm{~B}$. This plane intersects with the rotational plane created by the sodium (in this case, the $\mathrm{Na}_{\text {III }}$ ). Where these cross will determine, approximately, the potential minimum (though the small in-plane barrier from other sodiums will shift this position slightly). One might expect from oxygen's greater electrostatic dipolar polarizability that the term $U_{\text {pol }}$ rivals $U_{\text {multipole }}$ in importance. While this is true in certain guest locations, it does not seem to be the case here. Because of oxygen's negative partial charge, $U_{\mathrm{pol}}$ and $U_{\text {quad }}$ agree in the sense of orientation they attempt to impose on $\mathrm{H}_{2}$, but the magnitude of $U_{\text {quad }}$ seems to be much more important at this location. With such close proximity to so many oxygen, the short-range repulsion is large in magnitude, but it does not have a strong dependence on orientation.

In summary, the adsorption site we have just discussed in some detail is one in which both $\mathrm{O}$ and $\mathrm{Na}$ atoms make notable contributions to the potential surface. There are certainly other locations where the situation is different [for example, those of Figs. 2(d) and 2(e) in part I]. But the site that we have discussed is most characteristic. It seems that, typically, $\mathrm{Na}$ will make the dominant contribution to the size and location of the potential maximum. This position may be slightly altered by $\mathrm{O}$ atoms, as one can see by looking from Fig. 1(a) to 1(b). Furthermore, when two cations (typically $\mathrm{Na}_{\text {II }}$ and $\mathrm{Na}_{\text {III }}$ ) are quite close (less than $3 \AA$ ) to the $\mathrm{H}_{2}$ guest, the crossing of their respective "planar rotor" planes can [as in Fig. 2(e) of part I] produce a librational minimum whose position is slightly altered by $\mathrm{O}$ anions. This minimum will determine the shape of the rotational wave function when the temperature is well below the height of the saddle point. On the other hand, when one considers other features of the potential surface like the height of the saddle point, its location, and the detailed morphology of the surface, it seems the exception rather than the rule that these are due to $\mathrm{Na}$ alone. We must conclude that it is the combination of the cationic and anionic potential surfaces that produces the structure typical of the majority locations where $\mathrm{H}_{2}$ is likely to adsorb at $50-300 \mathrm{~K}$. (Statistics on the barrier to rotation along 50 and $300 \mathrm{~K}$ trajectories may be seen in Fig. 3 of part I.)

Because our technique involves solving the angular Schrodinger equation on an angular grid, we obtain the shape of the rotational wave function of hydrogen, $\Psi_{n}(\Omega)$ for the first few eigenstates: $n=1, \ldots 9$. This allows us to determine to what extent the molecule is "pinned" at a single orientation, and to see how the shape of the orbital is correlated with the host environment. The limited rotational freedom available to a guest molecule may explain why specific reaction mechanisms are enhanced by a zeolite catalyst. Most theoretical studies, our own included, attempt to decouple rotational from other motions. Including all of these motions in a single quantum chemical calculation is a formidable challenge. One might argue that rotations may be ignored based on the high potentential, energy barrier that hinders the rotor. ${ }^{39}$ Yet the low rotational inertia of $\mathrm{H}_{2}$ may render it fairly delocalized, even in a strongly hindering potential, and this possibility is one that our calculation seeks to explore.
Along with the potential energy contours in Fig. 2, we have also indicated the orientation of the hydrogenic bond axis,

$$
\hat{n}=\langle\Psi|\Omega| \Psi\rangle .
$$

The ground and first excited states are both oriented as one would naively expect. In particular, the first excited state has $\hat{n}$ aligned, within our ability to resolve, with the minimum of the angular potential energy surface. The ground state $\hat{n}$ is shifted slightly toward the saddle point. The second excited state and several of the higher-lying states have values of $\hat{n}$ which bear no obvious relationship to the features of the surface; perhaps this is inevitable, given that the orientational wave functions must be mutually orthogonal. Other excited states (e.g., the third) again have values of $\hat{n}$ which are approximately aligned with the minimum-energy direction.

When the temperature is much less than the saddle-point barrier (on the order of $350 \mathrm{~K}$ for the model we study), the uncertainty in orientation is determined by the extent of the wave function, and reorientation between equivalent wells occurs by tunneling. The coherent tunneling frequency is determined from the tunnel-splitting energy; typically around $30 \mathrm{~cm}^{-1}$, corresponding to $1 \mathrm{ps}^{-1}$. We will return to this in Secs. III C and $\mathrm{E}$ on neutron scattering below.

The top image in Fig. 3(a) is a view of the potential surface for reference. The center and bottom images show the probability-density, $|\langle\Psi(\Omega) \mid \Psi(\Omega)\rangle|^{2}$, for the ground and first excited states, respectively, for the $10 \mathrm{~K}$ position discussed above $($ black $/$ white $=$ high/low probability). One can see the nodal line for the first excited state, which is absent for the ground state. The maxima and minima of $|\langle\Psi(\Omega) \mid \Psi(\Omega)\rangle|^{2}$ coincide with the potential minima and maxima.

Figure 3(b) is an alternate view of the ground state data, with spherical coordinates represented along Cartesian axes. (The two peaks, corresponding to the exchange symmetry of $\mathrm{H}_{2}$, are, in principle, identical. Small discrepancies are a result of the finite computational grid size.) For an absorption site of interest, one might ask about the size of the solid angle that contains most of the probability density. If the solid angle is small, $\mathrm{H}_{2}$ is firmly pinned by the potential. In catalysis applications, where the accessibility of hydrogen to reagent molecules is of interest, the range of probable hydrogenic orientations would give one a sense of whether a particular reagent can approach and interact in the required way. The darker regions contain $90 \%$ of the probability density, and these encompass an angular region of approximately 7.6 steradians. This is not a small angle, and hence not a situation where the guest is pinned particularly firmly by the potential. While the guest avoids the potential maximum, an orientation that has it approximately end-on to a $\mathrm{Na}_{\mathrm{II}}$ cation, $\mathrm{H}_{2}$ is free to librate over roughly $60 \%$ of angle space.

The location of hydrogen in one of the supercage doorways is somewhat special, in the sense that cage doorways can be viewed as "transition states" that must be traversed in order for guests to diffuse through zeolites. Experiments and calculations have revolved around the rate at which hydrogen diffuses in zeolite $\mathrm{A},{ }^{13,43}$ so the nature of this transition state, and the barrier, if any, which the doorway pro- 

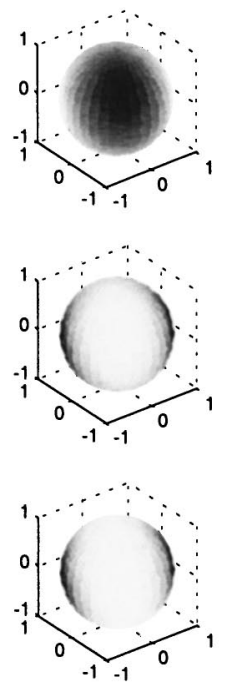

(a)

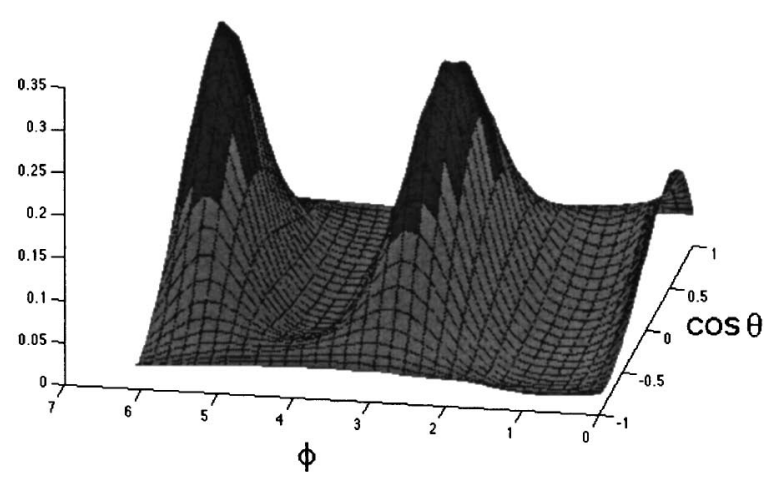

(b)

FIG. 3. (a) Potential surface (top), modulus squared of ground state (middle), and first excited state (bottom) wave functions for the $10 \mathrm{~K}$ position discussed in text. Darker regions are higher in magnitude. (b) Second view of modulus squared of ground state wave function [middle image of (a)]. Wave function is normalized, and plot as a function of spherical coordinates $\phi$ and $\cos (\theta)$. Darker regions represent area whose probability density integrates to $90 \%$ of total probability.

vides to activated transport is of interest. We have equilibrated hydrogen at $50 \mathrm{~K}$, restricting its position to the plane of one of the doorways (on the A side of $\mathrm{Na}_{\mathrm{III}}$ ). We have noted the following features. The separation factor is approximately 3.9 which is in the range expected at locations on this side of $\mathrm{Na}_{\text {III }}$. The average tunnel-splitting energy is unremarkable as well: $43 \mathrm{~K}$. The average energy, $-1350 \mathrm{~K}$, is slightly higher than the average energy of $-1410 \mathrm{~K}$ for unconstrained trajectories at $50 \mathrm{~K}$. So one may view the passage through the doorway as activated transport at very low temperatures; though this is evidently not the major activating event requiring $450 \mathrm{~K}$ that is predicted by Cohen de Lara and co-workers. ${ }^{43}$ This lack of appreciable barrier height associated with the doorways is consistent with the analysis of neutron scattering data, ${ }^{13}$ showing that an isotropic jump model with jump length $3.9 \AA$ (much smaller than the cage-to-cage distance) fits best to the data. Thus jumps through the doorways of $\mathrm{Na}-\mathrm{A}$, whose free diameter is roughly $2.9 \AA$, seem to play no dramatic role in limiting transport.

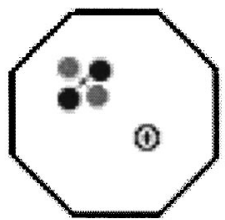

(a)

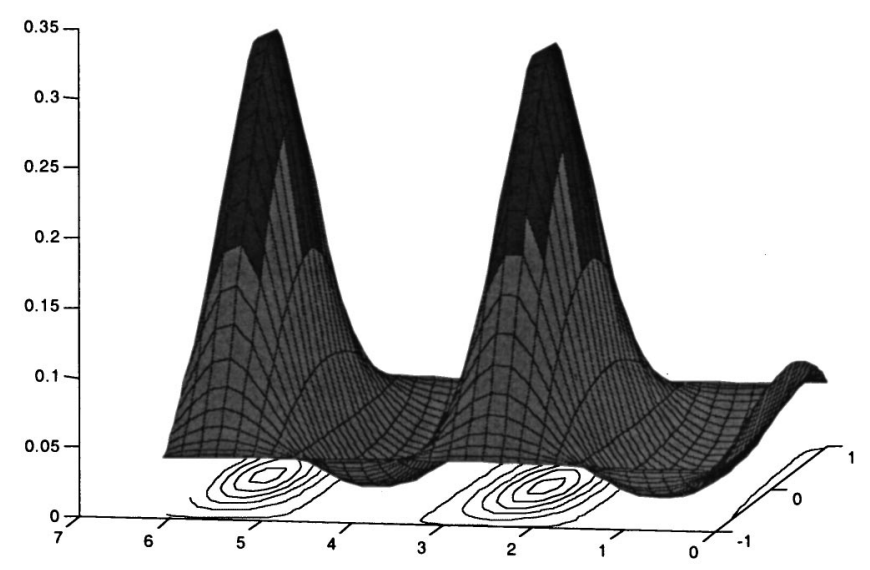

(b)

FIG. 4. (a) Schematic view of hydrogen within the doorway of Na-A. At 50 $\mathrm{K}$, hydrogen's center-of-mass is free to move within a range of approximately $0.1 \AA$ from its minimum position, which located approximately $1 \AA$ from the center of the doorway, and diagonally across from $\mathrm{Na}_{\text {II }}$. Its most (least) favorable orientations are shown as dark gray (light gray) images. (b) Modulus squared of ground state wave function for hydrogen in a typical location in doorway at $50 \mathrm{~K}$. Coordinates and color map as in Fig. 3(b).

As Fig. 4(a) indicates, hydrogen will be situated in the doorway so that it is diagonally across from the $\mathrm{Na}_{\mathrm{II}}$. Its preferential orientation is side-on to this cation, and within the plane of the doorway. This is not surprising, as it takes advantage of electrostatic interactions with both sodium and the doorway oxygens. One finds, though, that hydrogen's orientation is not highly constrained. An analysis like the one for Fig. 3(b) produces Fig. 4(b). Hydrogen has 90\% of its probability within 7.8 steradians. While it avoids being end-on to the cation, it is free to liberate in the plane of the doorway with an amplitude of around $60^{\circ}$. Furthermore, the potential varies slowly for rotations out of the doorway. So long as hydrogen remains side-on to the cation, it is free to rotate by more than $120^{\circ}$ about the doorway plane.

\section{B. Heat of adsorption, dependencies on model parameters}

The enthalpy, or heat, of adsorption for our model hydrogen in $\mathrm{Na}-\mathrm{A}$ may be compared with that of Basmadjian. ${ }^{44}$ This author measured adsorption equilibria and separation factors for hydrogen and deuterium in a variety of porous solids, in the temperature range of 75-90 K. While data on zeolite $\mathrm{Na}-\mathrm{A}$ are given, the zeolite was apparently in the hydrated from. We might expect these experiments to give a lower heat of adsorption than would our model of a dehydrated zeolite, ${ }^{45}$ and indeed his value of $\Delta H \equiv H_{g}-H_{s}=7.5 \mathrm{~kJ} /$ mole (his Fig. 4 extrapolated to zero coverage) falls slightly below our estimate given below, taken at $80 \mathrm{~K}$. 
For guest hydrogen in the low coverage limit, the average potential energy, $\bar{\phi},{ }^{46}$ results from guest-host interactions alone. In the adsorbed phase, the total energy will then be a sum of this potential energy and the kinetic contribution to the internal energy. To calculate $H_{g}$, the enthalpy in the gas, we need the internal energy for a freely-rotating hydrogen molecule, obtained as ${ }^{18}$

$$
\begin{aligned}
U_{g}= & \frac{1}{Z}\left(\Sigma_{j \text { even }} B j(j+1)(2 j+1) e^{-B_{j}(j+1) / k T}\right. \\
& \left.+\Sigma_{j \text { odd }} 3 B j(j+1)(2 j+1) e^{-B j(j+1) / k T}\right)
\end{aligned}
$$

with

$$
\begin{aligned}
Z= & \Sigma_{j \text { even }}(2 j+1) e^{-B j(j+1) / k T} \\
& +\Sigma_{j \text { odd }} 3(2 j+1) e^{-B j(j+1) / k T} .
\end{aligned}
$$

At $80 \mathrm{~K}$, this value is $U_{g}=90 \mathrm{~K}$, slightly more than the classical equipartition value of RT for a linear rotor. One assumes that the translational modes are populated according to equipartition, so that the enthalpy for free hydrogen at 80 $\mathrm{K}$ is

$$
H_{g}=U_{g}+\mathrm{RT}=290 \mathrm{~K} .
$$

Our MC calculation yields an average energy of $-1360 \mathrm{~K}$, which is a sum of rotational kinetic and potential energy. It is unclear whether one ought to calculate the enthalpy of the adsorbed phase as if it were mobile in the manner of a gas or highly localized (most likely, something in-between; the experimental isotherms are somewhat equivocal ${ }^{44}$ ). Thus one might try the form

$$
H_{s}=-1360 \mathrm{~K}+f \mathrm{RT},
$$

where $f$ is a parameter between $3 / 2$ (freely translating in three dimensions) and 3 (confinement in a three dimensional well). Thus our prediction for $\Delta H=H_{g}-H_{s}$ falls in the neighborhood of $\Delta H \approx 12 \mathrm{~kJ} / \mathrm{mole}$. Larin and Cohen De Lara ${ }^{38}$ have made a recent calculation of $\mathrm{H}_{2}$, with orientation fixed at its minimum-energy configuration, and estimate this heat in the range of $8-12 \mathrm{~kJ} / \mathrm{mole}$. For further comparison, quantum chemical calculations give several estimates of the binding of hydrogen in its lowest-energy configuration near a single sodium cation. Depending on method, these fall in the range of $\bar{\phi}=7.6-12.1 \mathrm{~kJ} / \mathrm{mole}^{5}{ }^{5} 12.0-12.8 \mathrm{~kJ} / \mathrm{mole},{ }^{4}$ and $12.8-$ $13.9 \mathrm{~kJ} / \mathrm{mole}^{42}$ with some earlier studies giving 11.5 $\mathrm{kJ} / \mathrm{mole}^{3}$ and $10.1 \mathrm{~kJ} / \mathrm{mole}^{47}$ If we look at MP2 result with a high quality basis set, Ref. 5 predicts a value of $12.1 \mathrm{~kJ} /$ mole; the still larger basis set in Ref. 42 produces the value $13.9 \mathrm{~kJ} / \mathrm{mole}$. The classical GCMC simulation of Ref. 15, taking into account polarization interactions, finds an internal energy of adsorption of $U=-18.3 \mathrm{~kJ} / \mathrm{mole}$, at the lowest densities and pressures of $\mathrm{H}_{2}$ studied.

The agreement of thermodynamic quantities with experiment is one test of potential parameters. Electrostatic charges, in particular, have a strong role in determining thermodynamic quantities like enthalpy and separation behavior, as well as spectral characteristics of guests. We have repeated some of our simulation runs taking electrostatic charges of $q_{\mathrm{Na}}=0.8 e ; q_{O}=-0.2 e$. The heat of adsorption at
$80 \mathrm{~K}$ rises to $\Delta H \approx 17 \mathrm{~kJ} /$ mole with these charges, which worsens agreement with experiment. The heights of in-plane barriers, and quantities which depend on them, are notably different for the two choices of charge. Barriers are higher by a factor of approximately 2, and separation factors are higher by factors of 1.1 and 1.3 for runs performed at 80 and $50 \mathrm{~K}$, respectively. Neutron tunnel-splitting peaks shift downward by more than $10 \mathrm{~cm}^{-1}$; we found a peak at $26 \mathrm{~cm}^{-1}$ at $80 \mathrm{~K}$ and $12 \mathrm{~cm}^{-1}$ at $50 \mathrm{~K}$.

We also checked the effect of strengthening the Lennard-Jones dispersion attraction [which also affects the short range repulsion, as in Eq. (6) of part I], to reflect the Kirkwood-Muller result cited in part I. Again, we found that there were changes in the mean energy of the adsorbed guest; at $135 \mathrm{~K}$ the energy went from $\bar{\phi}=-800 \mathrm{~K}$ to $\bar{\phi}$ $=-1400 \mathrm{~K}$. This is expected, given that the attraction for both $\mathrm{Na}$ and $\mathrm{O}$ atoms is almost twice as strong for this new parametrization. Over the course of several runs with the new parametrization, the tunnel-splitting energy decreased by only a bit, from 38 to $35 \mathrm{~cm}^{-1}$. The separation factor increased by a factor of less than $1 \%$, which was not significant.

\section{Energy density of states and simulated neutron scattering spectrum}

Neutron scattering techniques are ideally suited for the study of the dynamics of hydrogen-containing guest molecules in zeolites, and especially for dynamics of molecular hydrogen. The principal reason for this is the more than one order of magnitude higher neutron scattering cross section of $\mathrm{H}$ compared with that of virtually any other element, which makes it possible for INS to be in fact a sensitive probe of the dynamics of adsorbed molecules despite the scattering from the zeolite framework, which, for example, dominates IR spectra below about $1000 \mathrm{~cm}^{-1}$.

The internal HH stretching mode becomes IR active in the $\mathrm{H}_{2}$ /zeolite system, and is most conveniently observed by that method. ${ }^{48}$ The five external modes of the molecule may be described as two hindered whole body rotational modes and three vibrational modes involving CM displacement. These may not be distinct modes depending on the shape of the respective potential energy surfaces.

A most important property of INS in this context is the fact that it is possible to measure the rotational tunnel splitting of the librational ground state of the $\mathrm{H}_{2}$ molecule. This transition is not directly observable by optical techniques because it requires a change in the total nuclear spin of the molecule. To put it another way, neutrons can interact with the nuclear spin of the molecule by virtue of the neutron nuclear spin, while photons cannot couple to the nuclear spin. Thus neutron scattering allows transitions between ortho- and parahydrogen to be probed. Reference 40 contains a derivation of the incoherent neutron scattering function, $S(\mathbf{q}, \omega)$ for a dilute phase of $\mathrm{H}_{2}$. In order to simulate inelastic neutron spectra, which are typically taken from samples with microcrystalites inclined at various angles, we average q over all crystallographic directions. In the course of this average, we truncate an expansion in $j_{n}^{2}(q d / 2)$ at second 


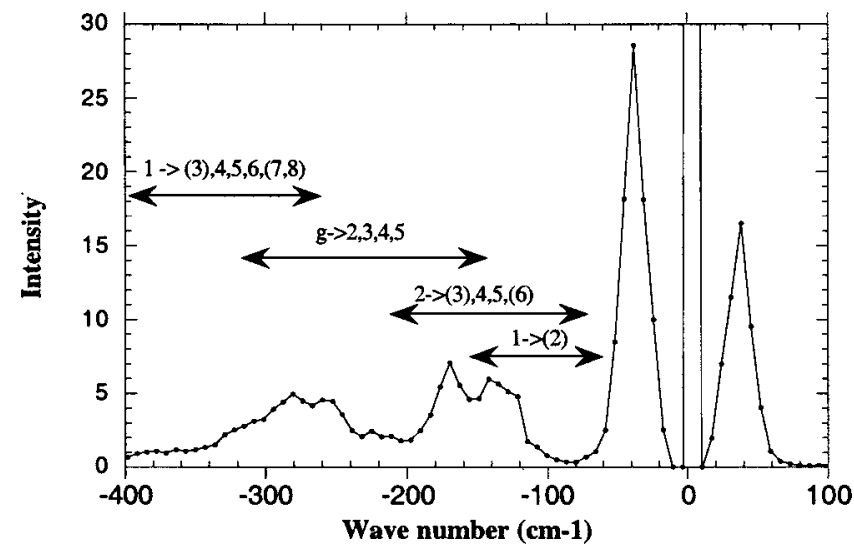

FIG. 5. Intensity (arbitrary units) as a function of neutron energy, as given by Eq. (12), for a $T=100$ trajectory. The large peaks centered at $\pm 38 \mathrm{~cm}^{-1}$ are the ground to first-excited state (tunnel-splitting) transitions. Other transitions produce bands as indicated; with states in parentheses making relatively minor contributions. " $g$ " denotes ground state.

order $(n=2)$. It follows that, as a rule, ortho-ortho transitions will scale with $j_{2}^{2}(q d / 2)$ and para-ortho transitions with $j_{1}^{2}(q d / 2)$. The smaller $q$ is, the greater will be the relative importance of para-ortho transitions. For $\mathrm{H}_{2}$, as we will see below, ground state to first-excited and ground state to second-excited state transitions are para-ortho; ground stateto-third are a mixture of para-ortho and ortho-ortho (determined by the location of $\mathrm{H}_{2}$ within the zeolite). So if one is able to control $q$, one can vary the contributions of the two types of transition to the scattering function. For example, features from ground to the two lowest-lying excited states will be more clearly visible at lower $q$, and the fraction of para states in the third excited state can be estimated as $q$ is varied. (It may also be determined by comparison with Raman spectroscopy, in which a transition to an ortho-thirdexcited state from the ground state is forbidden by a selection rule.)

Figure 5 is a "stick" spectrum, showing a momentumaveraged incoherent scattering function $S(\omega)$ for a 2000 step, $100 \mathrm{~K}$ simulation, calculated as

$$
\begin{aligned}
& S(\omega)=\sum_{I} \rho_{I}\left[\frac { 1 } { 2 } \sum _ { i } ^ { \text { ortho } } \rho _ { i } \sum _ { f } ^ { \text { ortho } } \left(\bar{j}_{0}^{2}(q d / 2) \delta(\omega)\right.\right. \\
& \left.+4 \pi \bar{j}_{2}^{2}(q d / 2) \sum_{m=-2}^{2}\left|\left\langle i\left|Y_{2}^{m}(\Omega)\right| f\right\rangle\right|^{2} \delta\left(\omega-\omega_{i f}\right)\right) \\
& +4 \pi \frac{1}{4} \sum_{i}^{\text {ortho }} \rho_{i} \sum_{f}^{\text {ortho }} \bar{j}_{1}^{2}(q d / 2) \sum_{m-1}^{1}\left|\left\langle i\left|Y_{1}^{m}(\Omega)\right| f\right\rangle\right|^{2} \\
& \times \delta\left(\omega-\omega_{i f}\right)+4 \pi \frac{3}{4} \sum_{i}^{\text {ortho }} \rho_{i} \sum_{f}^{\text {ortho }} \bar{j}_{1}^{2}(q d / 2) \\
& \left.\times \sum_{m-1}^{1}\left|\left\langle i\left|Y_{1}^{m}(\Omega)\right| f\right\rangle\right|^{2} \delta\left(\omega-\omega_{i f}\right)\right] .
\end{aligned}
$$

The precursor to Eq. (12) can be found in Ref. 40, where it is noted as $S_{\phi}$; Eq. (12) has, as an additional step, an average over wave numbers $q$, as discussed below. $d$ is the

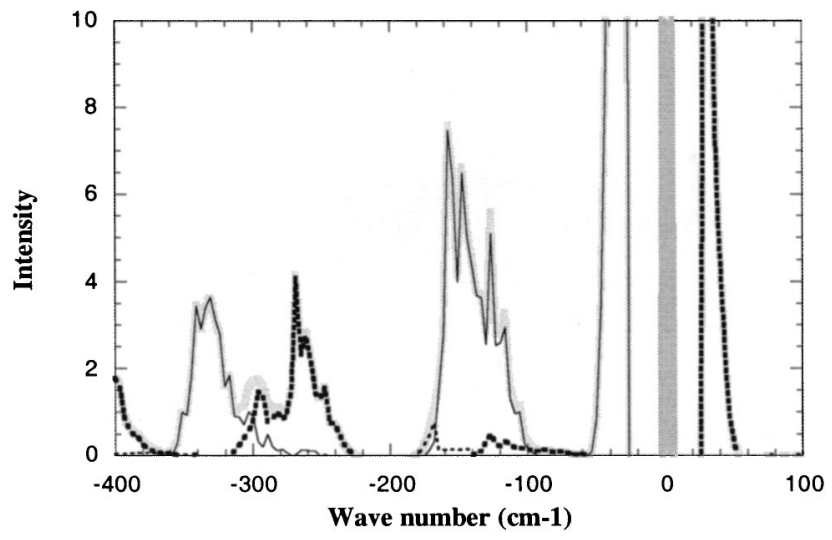

FIG. 6. Neutron intensity plotted as in Fig. 5, corresponding to a 2000 step trajectory at $50 \mathrm{~K}$ on the $\mathrm{A}$ side of the $\mathrm{Na}_{\text {III }}$ ion. Solid, heavy gray line: contributions from all transitions. Thin line: transitions from the ground state to the first, second, and fourth-and-higher excited states. (For this trajectory, the third state was almost exclusively para.) Heavy-dashed line: transitions from the first excited state to the ground (positive wave number); the second (around $100 \mathrm{~cm}^{-1}$ ); and the third, fourth, fifth (around 275 $\mathrm{cm}^{-1}$ ), and sixth and higher excited states (above $350 \mathrm{~cm}^{-1}$ ). Thin-dashed line: transitions from the second to the third (around $150 \mathrm{~cm}^{-1}$ ) and higher excited states.

length of the $\mathrm{H}_{2}$ bond (unless otherwise mentioned, $d=d_{0}$ $=0.77 \AA$ in our simulation). The density of initial states is

$$
\rho_{i}=\frac{g_{i} e^{-\beta E_{i}}}{\sum_{k} g_{k} e^{-\beta E_{k}}} .
$$

The spectrum in Fig. 5 contains contributions from various center-of-mass positions $\mathbf{R}$; this is a consequence of the sum over initial states $I$, which from Eq. (4) implies a sum over center-of-mass locations. (The trajectory used for Fig. 5 is displayed in Fig. 9 of part I.) In addition, we have used averaged values of spherical bessel functions, $\bar{j}_{n}^{2}(q d / 2)$; averaged uniformly over wave numbers $q$ between 1.7 to 4.6 $\AA^{-1}$. ${ }^{4}$ This choice was made in order to reflect the range of wave numbers used in a typical measurement, that of Nicol et al. ${ }^{29}$ and it was made independently of the experimental data discussed below (Sec. III D). Figure 5 is annotated to show the initial and final states which dominate various bands in the spectrum. Because we have assumed a thermal distribution of para- and ortho-states (not always true in a neutron scattering experiment at low temperature, due to the slow rate of spin conversion in some hosts), excited states give rise to much less noticable features on the energy-loss side of the spectrum. The contribution of the tunnel-splitting transition is indicated on the energy-gain side with darker bars. This contribution occurs at a mean energy of $38 \mathrm{~cm}^{-1}$. The width (FWHM) of approximately $20 \mathrm{~cm}^{-1}$ arises from, of course, the various of positions assumed during the MC walk (Fig. 9 of part I) for this static calculation.

At a temperature of $50 \mathrm{~K}$, we find that the neutron peak around the tunnel-splitting energy becomes quite narrow and markedly asymmetrical, making it difficult to assign a FWHM (the width from the peak to the half-maximum on one side is about $15 \mathrm{~cm}^{-1}$ ). The position of the peak is somewhat reduced. It falls at $31 \mathrm{~cm}^{-1}$ for the run of Fig. 6, and averages of several independent runs on the " $\mathrm{A}$ " and " $\mathrm{B}$ ", 
sides give slightly different answers: $32 \pm 3$ and 25 $\pm 3 \mathrm{~cm}^{-1}$, respectively. Agreement with the $50 \mathrm{~K}$ experiments, described in Sec. III D below, is quite good.

In comparing Figs. 5 and 6, one sees that the ratio of the tunnel-splitting peak height to the height of the broad, featured band at higher energies, is slightly greater at the lower temperature. Also, the peak regions within the broad band become better resolved. Because of this better resolution, it is informative to indicate in the figure which regions of the spectrum arise from which rotational transitions. Transitions beginning in the ground, first, and second excited states are shown with solid thin lines, dashed heavy lines, and dashed thin lines, respectively. The total spectrum is given as a heavy gray line. A band centered at around $150 \mathrm{~cm}^{-1}$ arises largely from transitions between the ground and secondexcited state, although the simulation predicts that transitions from the first to the second, and from the second to the third make small and nonoverlapping contributions. Contributions from transitions between the first and the third, fourth, and fifth excited states merge to form a band. The peak at 270 $\mathrm{cm}^{-1}$ is due to transitions from first to third, and transitions from first to fourth and fifth overlap to create the secondary peak at around $390 \mathrm{~cm}^{-1}$. (The peak at around $400 \mathrm{~cm}^{-1}$ comes from transitions between first and the sixth excited state.) Another, distinguishable, band comes from transitions between ground and third and higher excited states; it peaks at around $330 \mathrm{~cm}^{-1}$.

Further insight can be gained by viewing the density of energy states (DOS) directly. In Figs. 7(a) and 7(b) we have shown the probability of finding a particular energy for each of the four lowest-lying states, for the 100 and $50 \mathrm{~K}$ runs described in Figs. 5 and 6. For example, the mean energy difference between the ground and first excited states in Figs. 7 (a) and 7(b) is 39 and $33 \mathrm{~cm}^{-1} ; 38$ and $31 \mathrm{~cm}^{-1}$ from the neutron spectra. Of course, the widths of two bands in Figs. 7 do not sum to produce the width of transition between them on the neutron spectrum. For one thing, Figs. 7 do not take into account the matrix elements in Eq. (12), which vary a bit from location-to-location in the crystal. More importantly, a location that features a low ground state energy will also feature a low first-excited state energy, and so on. Since transitions are modeled to occur between states at a single location, we expect the width of any peak in the neutron spectrum to be smaller than the combined widths of the two bands in Figs. 7 that produce it. This is apparent from the approximate widths of the bands, which are $125 \mathrm{~cm}^{-1}$ for the first three states and $175 \mathrm{~cm}^{-1}$ for the fourth in Fig. 7(a), and approximately $90 \mathrm{~cm}^{-1}$ for all four states in Fig. 7(b). The density of energy states are asymmetric with high-energy tails. It is tempting to associate this asymmetry with those in, e.g., the tunnel-splitting or the ground-to-second excited state peaks in the $50 \mathrm{~K}$ neutron spectrum. However, there does not seem to be an obvious connection between the two. Large $\left(>37 \mathrm{~cm}^{-1}\right)$ neutron energy transfers occur between states at all parts of the DOS curves. The asymmetry may simply reflect the fact that, given there is a limit to the height of the in-plane barrier, there is a limit to the proximity of the ground and first excited state, which seems to be around 28 $\mathrm{cm}^{-1}$ for $50 \mathrm{~K}$ data. There is less of an asymmetry in the 100

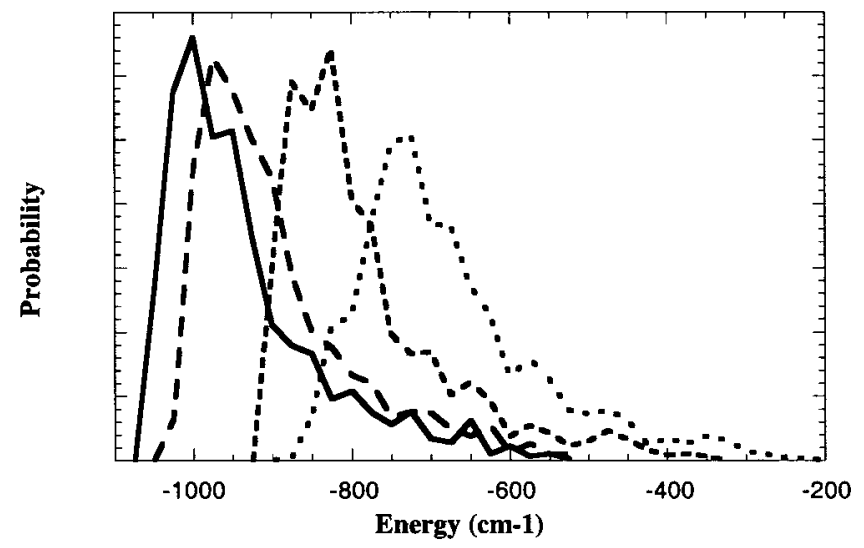

(a)

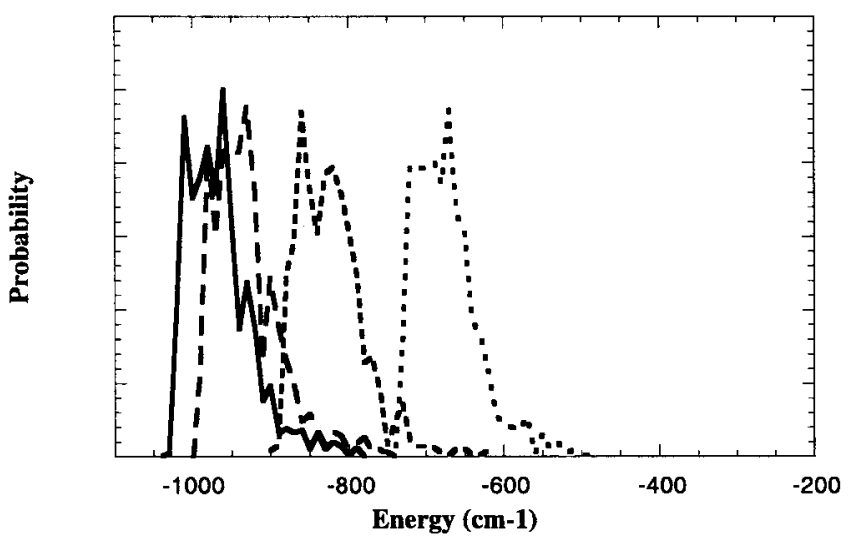

(b)

FIG. 7. Density of energy states for 2000 step trajectories. Solid, dashed, finer dashed and dashed lines correspond to the ground through the third excited states. (a) Trajectory (Fig. 5) at $100 \mathrm{~K}$. The four bands have mean energies of $-1338,-1282,-1117$, and $-963 \mathrm{~K}$, respectively. (b) Trajectory (Fig. 6) at $50 \mathrm{~K}$. The four bands have mean energies of $-1390,-1342$, -1186 , and $-970 \mathrm{~K}$, respectively.

$\mathrm{K}$ neutron tunnel-splitting peak, and the closest that the ground and first excited states approach in energy is a mere $14 \mathrm{~cm}^{-1}$. One might speculate that at the higher temperature, the hydrogen can have a small number of energetically unfavorable, close approaches to host atoms, yielding large rotational barriers and filling in this low-energy side of the neutron tunnel-splitting peak.

The $10 \mathrm{~K}$ trajectory yields the neutron spectrum of Fig. 8. Peaks are centered on 30,150 , and $340 \mathrm{~cm}^{-1}$ for transitions from ground to the first, second, and fifth excited states. There are no visible transitions between ground and third or fourth because, for this set of locations in the host, both of these states are always para. That is, the symmetries of the states read as follows: $p, o, o, p, p, o \ldots$ as one goes up in energy. The width from tunnel-splitting peak to half max is very narrow: $4 \mathrm{~cm}^{-1}$. The peak falls between 30 and 31 $\mathrm{cm}^{-1}$, as does the mean of the distribution of tunnel-splitting energies. The ground and first excited states have a minimum proximity of $29 \mathrm{~cm}^{-1}$, very much like the situation at $50 \mathrm{~K}$.

\section{Experimental details}

Inelastic neutron scattering measurements were carried out on the thermal neutron time-of-flight spectrometer IN4 at 


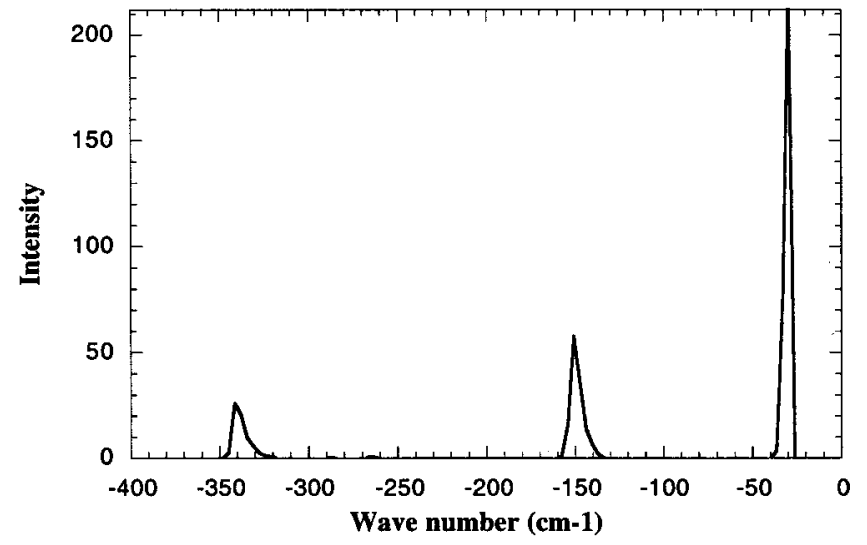

FIG. 8. Neutron intensity plotted as in Fig. 5, corresponding to the 2000 step trajectory at $10 \mathrm{~K}$ seen in Fig. 8 of part $\mathrm{I}$.

the Institut Laue-Langevin (Grenoble, France). This instrument provides a pulsed monochromatic beam of neutrons and scans the energies of the scattered neutrons by their time-of-flight. Most of the data was collected with an incident energy of $139 \mathrm{~cm}^{-1}$. The energy resolution under this condition was $8 \mathrm{~cm}^{-1}$ (FWHM). Some additional data was collected with an incident energy of $557 \mathrm{~cm}^{-1}$ (resolution of $40 \mathrm{~cm}^{-1}$ FWHM). Following data collection on the bare sample of approximately $6 \mathrm{~g}$ of fully dehydrated zeolite $\mathrm{Na}-\mathrm{A}$ it was loaded in situ at $85 \mathrm{~K}$ with 1 molecule per supercage of $\mathrm{H}_{2}$ and subsequently annealed at $100 \mathrm{~K}$.

\section{E. Experimental results and comparison with computation}

Because the inelastic peaks were barely resolved from the elastic peak, we show in Fig. 9 the difference spectra (for $E_{i}=139 \mathrm{~cm}^{-1}$ ) of the $\mathrm{H}_{2}$ loaded $\mathrm{Na}-\mathrm{A}$ with the bare zeolite at temperatures of 5 and $50 \mathrm{~K}$. The figure shows the sum of approximately 30 detector banks at the largest scattering angles, because the rotational form factor for $\mathrm{H}_{2}$ increases with scattering angle $(Q)$ over the kinematic range of this experiment. The $5 \mathrm{~K}$ spectrum shows a pair of broad transitions at an energy of $26.6 \mathrm{~cm}^{-1}$ with a FWHM of $18 \mathrm{~cm}^{-1}$, as well as a weaker peak at about $70 \mathrm{~cm}^{-1}$ which is only

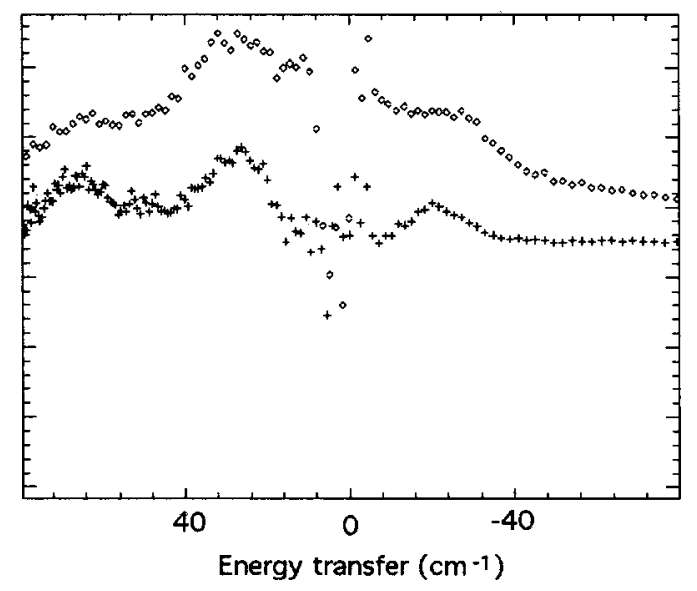

FIG. 9. Experimental INS spectrum of $\mathrm{H}_{2}$ in $\mathrm{Na}-\mathrm{A}$. Plus signs: $5 \mathrm{~K}$; Diamonds: $50 \mathrm{~K}$. (Origins are displaced vertically for visibility of both curves.)

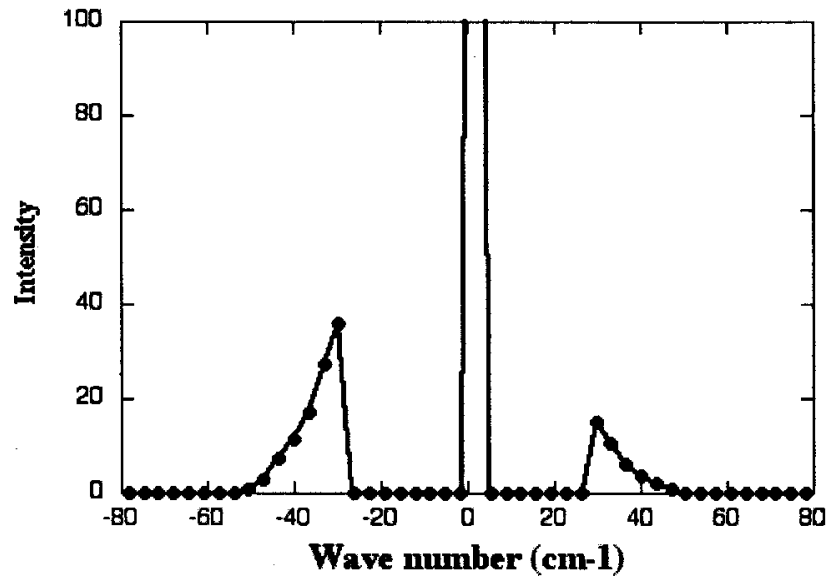

FIG. 10. Data from Fig. 6 for comparison with experiment.

observable by neutron energy loss. The pair of transitions shifts to slightly lower energy at $50 \mathrm{~K}$ and the width of these bands increases to $28 \mathrm{~cm}^{-1}$. In addition, a broad quasielasticlike component is evident under the inelastic transitions.

The distributions of ortho-para rotational transitions obtained from our computational studies (e.g., Fig. 6) at $50 \mathrm{~K}$ have peaks in the range of $22-35 \mathrm{~cm}^{-1}$ (depending on the simulation run). These are in remarkable agreement with the experimental data which was originally obtained independently. Figure 10 is a subset of the $50 \mathrm{~K}$ data of Fig. 6, plotted on the same scale as the experimental results to facilitate comparison.

Some sources of broadening are present in the experimental data and absent in the simulations. (see, for example, the discussion of decoupling of CM dynamics in Ref. 40.) One should note that the experimental data are broadened by an approximately Gaussian spectrometer resolution function which reduces the degree of asymmetry in the data. Nonetheless one can see some assymmetry in the peaks of Fig. 9, with more gradual trailing-off of intensity at higher energy transfers, in agreement with the simulated data. A second, somewhat weaker peak in the experimental data is visible in the neutron energy-loss side of the $5 \mathrm{~K}$ experimental spectrum, and may be present in the $50 \mathrm{~K}$ data as well. While the origin of this peak at about $70 \mathrm{~cm}^{-1}$ is not clear from the present data, there are essentially two possibilities. First, we note that this band occurs only in neutron energy loss, i.e., corresponds to an excitation from the ground state. This could suggest that this band be associated with a CM translation, since a vibrational state at $70 \mathrm{~cm}^{-1}(\approx 92 \mathrm{~K})$ would be depopulated at $5 \mathrm{~K}$. Rotational states, on the other hand, normally convert slowly, as we discussed above, because of the requirement that nuclear spins have to be flipped. By the same token, spin conversion has been observed in some cases for $\mathrm{H}_{2}$ in zeolites, and we cannot completely rule out the possibility that some $\mathrm{H}_{2}$ molecules have indeed reverted to the ground state at a certain adsorption site. While this seems highly unlikely, it would be consistent with our previous work on $\mathrm{H}_{2}$ in $\mathrm{CaNa}-\mathrm{A}^{29,50}$ and $\mathrm{ZnNa}-\mathrm{A}{ }^{51}$

At $50 \mathrm{~K}$ a very broad, diffuse background is apparent below the broadened rotational tunneling peaks. While this could arise in part from a broadening and concomitant popu- 
lation of the upper level for the $70 \mathrm{~cm}^{-1}$ mode, we suggest that this may in fact arise from very fast rotational diffusion of the hydrogen molecules as they move from site to site. In the course of this translational diffusion the molecules may experience a wide range of hindering potentials, which will give rise to a broad quasielastic line centered at $E=0$ and extending to as high an energy as $15 \mathrm{meV}$ (no barrier), while in between steps they are subject to the sort of well-defined potential described herein with associated rotational transitions. Translational diffusion of the hydrogen molecule in $\mathrm{Na}-\mathrm{A}$ was in fact described by Cohen de Lara and collaborators ${ }^{13}$ who found it, as would be expected, to be considerably slower than the rotational diffusion we apparently observe.

These results for $\mathrm{H}_{2}$ in Na-A may be compared with some previous INS studies, most notably that for $\mathrm{Na}-\mathrm{X}^{31}$ This is analogous to the way that several IR studies have attempted to relate the observed shift in $\nu(\mathrm{HH})$ to the basicity of the framework, i.e., the $\mathrm{Si} / \mathrm{Al}$ ratio of the zeolite. ${ }^{25-28}$ The INS spectra for a sample with just one $\mathrm{H}_{2}$ per supercage of $\mathrm{Na}-\mathrm{X}$ show the rotational tunneling transition at $18 \mathrm{~cm}^{-1}$, roughly half of that in $\mathrm{Na}-\mathrm{A}$. For a comparison of the barrier heights in $\mathrm{Na}-\mathrm{A}$ and $\mathrm{Na}-\mathrm{X}$, we utilize the simple doubleminimum potential with just one twofold barrier from our earlier work, rather than the form used here with separate barriers for in-plane and out-of-plane hindering. (The barrier heights obtained with the different models are not directly comparable.) With this model the barriers to rotation are 7.8 $\mathrm{kJ} / \mathrm{mole}$ for $\mathrm{H}_{2}$ in $\mathrm{Na}-\mathrm{X}$ and $6.4 \mathrm{~kJ} / \mathrm{mole}$ in $\mathrm{Na}-\mathrm{A}$. Kazansky and others, however, report a smaller shift in $\mathrm{Na}-\mathrm{X}$ for $\nu(\mathrm{HH})$ relative to free $\mathrm{H}_{2}$ : it is approximately $56 \mathrm{~cm}^{-1}$ in $\mathrm{Na}-\mathrm{X}$ and $90-100 \mathrm{~cm}^{-1}$ in $\mathrm{Na}-\mathrm{A}$. Thus, despite the higher barrier to rotation in $\mathrm{Na}-\mathrm{X}$ suggested by the INS result, the shift in $\nu(\mathrm{HH})$ is less in $\mathrm{Na}-\mathrm{X}$ than in $\mathrm{Na}-\mathrm{A}$. This comparison suggests that parts of the interaction affect the two observables to different degrees. The details of this should be accessible from theoretical efforts, such as the work of Larin and coworkers which is discussed below. And for example, Cohen de Lara has recently demonstrated how $\nu(\mathrm{HH})$ for an adsorbate depends on its orientation relative to the cation. ${ }^{52}$

Though the rotational hindering potential of Evett-Sams-MacRury, ${ }^{53}$

$$
V(\theta, \phi)=\lambda \cos ^{2}(\theta)+\mu(1-\cos n \phi),
$$

has been used by many authors to explain experimental results, it is important to keep in mind that agreement is only approximate between the eigenenergies for this potential and other similar potentials, including those we find in the actual model zeolite. For example, we have selected a typical location in the zeolite: $(2.177,-1.241,3.911) \AA$. [This location can be found on the trajectory of Fig. 6(a) of part I, and our motivation for selecting it is apparent in the paragraph below.] This location presents an in-plane barrier of 3.2 B, and an out-of-plane barrier of $7.3 \mathrm{~B}$ to the hydrogen guest. Transitions from the ground to the first few excited states can be calculated for this atomistic zeolite potential, and also for the potential of Eq. (14) for the corresponding model parameters: $\mu=1.6 \mathrm{~B}$ and $\lambda=4.1 \mathrm{~B}(=7.3 \mathrm{~B}-3.2 \mathrm{~B})$. Results are
TABLE I. Comparison of transition energies (in units of B) for various potentials.

\begin{tabular}{cccc}
\hline \hline Potential model & $g \rightarrow 1$ & $g \rightarrow 2$ & $g \rightarrow 3$ \\
\hline Atomistic zeolite & 0.92 & 2.3 & 3.8 \\
Eq. (14) & 0.95 & 2.5 & 3.3 \\
Eq. (15) & 0.94 & 2.3 & 3.8 \\
\hline \hline
\end{tabular}

shown in Table I. One can see that the agreement is good, but not as good as one would like, given the typical resolution of INS and IR experiments.

Also in Table I are the results of a different twoparameter model for the hindering potential, for the case $n$ $=2$ :

$$
V(\theta, \phi)=\lambda \cos ^{2}(\theta)+\mu(1-\cos 2 \phi) \sin ^{2}(\theta) .
$$

Equation (15) is perhaps an even more reasonable fit to the sorts of potential surfaces one sees from the atomistic zeolite model. The appropriate parameter values to match the inand out-of-plane barriers are $\mu=1.6 \mathrm{~B}$ and $\lambda=7.3 \mathrm{~B}$. As Table I shows, Eq. (15) reproduces the simulated spectrum more closely. Figure 11 contains contour maps of the rotational potentials. The good match between the atomistic potential [Fig. 11(a)] and Eq. (15) [Fig. 11(c)] is evident. As was noted in paper I, and is evident in Fig. 11(b), the rapid variation in Eq. (14) near $\cos (\theta)= \pm 1$ is nonphysical.

The sensitivity of eigenstate energies to the potential model makes the interpretation of experiment somewhat subtle. A case-in-point may possibly exist in the work of Braid et al. ${ }^{34}$ In their INS study of partially $\mathrm{Zn}$ exchanged zeolite A, bands were observed at 1.2 and $2.5 \mathrm{~B}$. These were fit to the solutions to the Schrödinger equation for Eq. (14) with $\lambda=\infty$ and $n=6$. One problem with the analysis was that no single value of $\mu$ fit both peaks. Additionally, the authors of Ref. 34 noted that the values of $\mu$ were very high when compared with a theoretical calculation, and also with experimental heats of adsorption. Finally, even though we
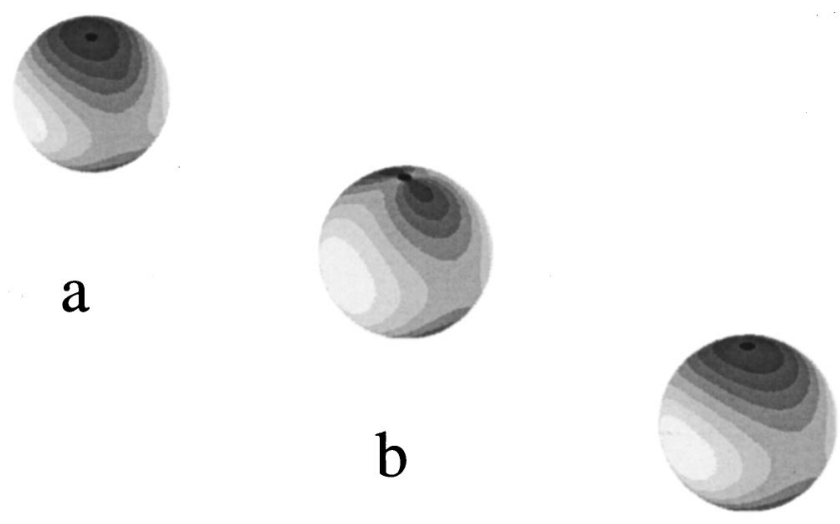

C

FIG. 11. (a) Contour map of the rotational potential energy corresponding to a typical $50 \mathrm{~K}$ location in $\mathrm{Na}-\mathrm{A}$. For comparison, the surface has been rotated so that the landmarks have approximately the same orientation as in (b) and (c). (b) Contour map corresponding to Eq. (14) with $\lambda, \mu$ chosen as 4.1 and $1.6 \mathrm{~B}$, to correspond to (a). (c) Contour map corresponding to Eq. (15) with $\lambda, \mu$ chosen as 7.3 and $1.6 \mathrm{~B}$, to correspond to (a). 
would expect the symmetry of the adsorbing potential to be higher for $\mathrm{Zn}$ substituted zeolite A than for $\mathrm{Na}-\mathrm{A}$, our simulations make us wonder whether $n \neq 2$ is typical in such a system. ${ }^{48}$ While we agree with Braid $e t$ al. that no choice of $\mu$, and seemingly, no choice of $\lambda, \mu$ in Eq. (14) will agree with experiment, we find that Eq. (15) gives the correct peak positions with $n=2$ when $\lambda=7.3 \mathrm{~B}$ and $\mu=1.6 \mathrm{~B}$. (This may not be a unique choice of parameters.) With this choice, the $g \rightarrow 1$ transition energy is $1.2 \mathrm{~B}$ and $g \rightarrow 2$ transition energy is $2.5 \mathrm{~B}$. One would expect these lines, corresponding to a para-to-ortho transition, to be strongly visible in the INS experiment. $^{54}$

\section{F. Comparison with IR experiment}

As mentioned above, several studies on $\mathrm{Na}-\mathrm{A}$ and other zeolites have related IR frequency shifts of $\nu(\mathrm{HH})$ to site occupancies. This type of association can be made when the IR band has two or more components, whose relative intensities may then be correlated with cation site populations according to the results from crystallographic studies. For example, two bands at 4075 and (a shoulder at) $4110 \mathrm{~cm}^{-1}$ have been observed ${ }^{25,27}$ for $\mathrm{H}_{2}$ in $\mathrm{Na}-\mathrm{A}$, whereas at lower pressures (one or less $\mathrm{H}_{2}$ per unit cell) only the former is evident. Other studies have located the shoulder at $4085^{23}$ or found higher-pressure data best fit with all three of these components. ${ }^{24}$ Based on cation site population arguments, Ref. 27 assigned the main $\nu(\mathrm{HH})$ band at $4075 \mathrm{~cm}^{-1}$ to $\mathrm{H}_{2}$ at $\mathrm{Na}_{\text {I }}$ and the second line either to $\mathrm{H}_{2}$ at $\mathrm{Na}_{\text {II }}$ or $\mathrm{Na}_{\text {III }}$, or a pressure-induced effect of having multiple hydrogens in a single cage. Since we find, however, that at low coverages $\mathrm{H}_{2}$ clearly prefers to be in the vicinity of $\mathrm{Na}_{\text {II }}$ and $\mathrm{Na}_{\text {III }}$, or $\mathrm{Na}_{\mathrm{I}}$ and $\mathrm{Na}_{\text {III }}$ (Figs. 5-9 of part I), we suggest that the above assignment be modified. There is some disagreement in the literature about the strengths of various adsorption sites, with Ref. 12 suggesting that adsorption in the window site (type II) is strongest, so that the main peak at $4077 \mathrm{~cm}^{-1}$ (their data) should be associated with that. On the other hand, the authors of references $6,13,37$, and 55 find that adsorption in the direction of $\mathrm{Na}_{\mathrm{I}}$, in closest proximity to $\mathrm{Na}_{\mathrm{III}}$, involves the deepest minimum, and that the frequency of oscillation in this well is approximately $200 \mathrm{~cm}^{-1} \cdot 36,38$ Our results emphasize the importance of the proximity of next-nearest neighbor cations, which can then give rise to distinguishable adsorption sites about the supposedly preferred cation. An argument in a similar vein can be found in Ref. 26, where the different aluminum content, hence basic strengths, of different six-membered rings are posited to produce different absorption bands.

Cationic disorder may make the attribution of these lines to adsorption sites more complicated. For example, it has been suggested ${ }^{56}$ that the overtone bands, which arise from the anharmonicity of the $\mathrm{H}_{2}$ vibration, may display shifts and widths that arise from a distribution of adsorptive sites. Such effects may also be evident in the INS spectra in our previous work, ${ }^{35}$ where structure in the rotational tunneling peaks is sometimes evident. A repeat of the present experiment with higher energy resolution would therefore be a highly desirable test of our simulation results.

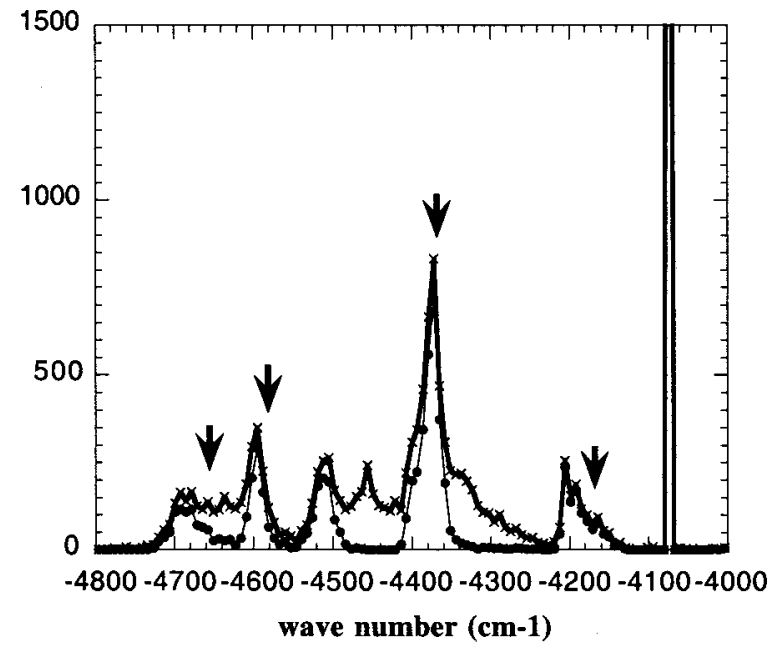

FIG. 12. Simulated line locations in IR spectrum of $\mathrm{H}_{2}$ in $\mathrm{Na}-\mathrm{A}$ at $50 \mathrm{~K}$. Filled circles: $\mathrm{H}_{2}$ located on A side of $\mathrm{Na}_{\text {III }}$ cation. Crosses: Superposition of spectra from $\mathrm{H}_{2}$ located on $\mathrm{A}$ and $\mathrm{B}$ sides of cation. Arrows note centers of experimental IR bands.

In the DRIFT work of Kazansky and collaborators, ${ }^{12}$ considerable numbers of weak satellites of the main $\nu(\mathrm{HH})$ vibrational band are observed. These could be either rotational or translational (CM mode) bands. Rotational transitions are subject to the optical selection rules that forbid transitions involving a change in the parity of the nuclear spin wave function of the molecule. In the low-coverage spectrum (Fig. 2 in Ref. 12) such satellites are observed at shifts, relative to $\nu(\mathrm{HH})$, of $93288 \mathrm{~cm}^{-1}$ and a doublet (or more) at $495-570 \mathrm{~cm}^{-1}$. Similar satellites were observed at roughly 100 and $290 \mathrm{~cm}^{-1}$ in DRIFT spectra of Beck and co-workers, ${ }^{23}$ and indeed a $90 \mathrm{~cm}^{-1}$ sideband was observed in the original FTIR study of this system. ${ }^{21}$ The lowest frequency band has been assigned by some workers to be a translational band and by others to a rotational band. Additionally, Kazansky and co-workers assign the remaining peaks to transitions to higher rotational states, using the freerotor states indexed by $J$ as a reference.

These observations can be addressed by our calculation. In Fig. 12 we have plotted the infrared lines that would appear for $\mathrm{H}_{2}$ bound on the A side (dots) and the sum of both the A and the B side (crosses), respectively. Only lines which are shifted upward in frequency are displayed in this figure.

These lines scale with a dipole intensity proportional to $|\langle i|\mu| f\rangle|^{2}$, where $i$ and $f$ denote vibrational, rotational, and electronic levels. Naturally, levels are expected to appear in the "vibrational" IR spectrum as a result of rovibrational coupling. The correct induced dipole moment matrix element would need to be calculated for each rovibrational transition. This was done long ago for freely-rotating hydrogen. ${ }^{20}$ The analogous calculation could, in principle, be done for the strongly hindered $\mathrm{H}_{2}$ system, but requires more than the rigid-rotor model of $\mathrm{H}_{2}$ in the current study. Thus the lines in Fig. 12 are weighted only by the usual Boltzmann factor for the likelihood of observing the initial rotational state $i$. In this figure, one finds that the A side rotational spectrum does a very good job in reproducing most of the lines seen by Kazansky and co-workers, the positions of which are marked by 
arrows. On the A side, the line centered at approximately $100 \mathrm{~cm}^{-1}$ corresponds to the $1\langle\leftrightarrow\rangle 2$ rotational transition; $290 \mathrm{~cm}^{-1}$ to both $g\langle\leftrightarrow\rangle 3$ transitions, which are mostly parato-para, and therefore forbidden in the incoherent neutron spectrum, and $1\langle\leftrightarrow\rangle 4$ transitions; and the ratio of lines between 430 and $610 \mathrm{~cm}^{-1}$ corresponds to $g\langle\leftrightarrow\rangle 5$ and higher state transitions). The B side spectrum has its $1\langle\leftrightarrow\rangle 2$ line shifted to a higher wave number, so that it merges with the A side peak at approximately $290 \mathrm{~cm}^{-1}$ and additional B side peaks at higher wave numbers create bands in the total spectrum. In the line at $288 \mathrm{~cm}^{-1}$ there is a small amount of first to third transition as well, but parity considerations suggest that it is not a strong contributor, since in most locations the third state is exclusively para; hence a first-to-third transition is IR forbidden. In summary, most features in the sum of A and B side data correlate with features in the experimental spectrum. We should emphasize that because $J$ is not a good quantum number and rotational levels are strongly perturbed in the zeolite, we would suggest that a transition like $J_{0 \rightarrow 2}$ does not provide an appropriate description. Nevertheless, as seen in Fig. 12, we find that transitions between the perturbed rotational states are good candidates for most of the IR lines seen in experiment.

There is a broad feature beneath the IR satellite shifted from $\nu(\mathrm{HH})$ by $93 \mathrm{~cm}^{-1}$. It is conceivable that this contains a CM mode, perhaps the self-same mode that appears in our INS spectrum as a second band near $70 \mathrm{~cm}^{-1}$ only on the neutron energy-loss side of the spectrum. Thus we might suppose that these IR satellites actually consist of two bands: one rotational and one $\mathrm{CM}$ in origin. As is indeed apparent in Fig. 2 of Ref. 12, this high-frequency satellite at $4170 \mathrm{~cm}^{-1}$ may well contain two features, with the second feature located at a slightly lower wave number, shifted by $67 \mathrm{~cm}^{-1}$ from $\nu(\mathrm{HH})$. In fact, in Fig. 3 of that reference, the two features, slightly shifted from their positions in Fig. 2 because of different hydrogen loading, are identified as distinct (Kazansky et al. attribute this second mode at higher coverages, to a second adsorption site at $\mathrm{Na}_{\mathrm{I}}$ ). Moreover, a second band appears also in the deuterium spectra in Figs. 6 and 7 of the same reference. The low-frequency member of this satellite pair for $\mathrm{H}_{2}$ is located at $4008 \mathrm{~cm}^{-1}$, which is $69 \mathrm{~cm}^{-1}$ redshifted from the main peak. The possible existence of a second, unresolved band could also explain the apparent asymmetry in this satellite.

As far as deuterium measurements are concerned, we note that two satellite features were observed for $D_{2}$ in $\mathrm{Na}-\mathrm{A} \cdot{ }^{19,21}$ The separation between them was quite large $\left(100 \mathrm{~cm}^{-1}\right)$; these sidebands were analyzed in terms of a rotational mode. Beck et al. ${ }^{23}$ also attributed a broad satellite observed to be shifted $100 \mathrm{~cm}^{-1}$ from the main peak to a rotational mode.

It is compelling to identify a broad feature at around 70 $\mathrm{cm}^{-1}$ from the main band in $\mathrm{Na}-\mathrm{A}$ as a $\mathrm{CM}$ mode and associate it with the band at about $50 \mathrm{~cm}^{-1}$ in the deuterium spectrum; i.e., shifted essentially by the mass isotope effect. ${ }^{12}$ To investigate the possibility of this mode being rotational rather than $\mathrm{CM}$ in origin, we have calculated energy levels for both deuterium and hydrogen, using a simplified hindering potential like Eq. (14) or, better, Eq. (15) (this type of analysis appears in Ref. 30), and have simulated hydrogen and deuterium using our atomistic zeolite potential at locations along a $50 \mathrm{~K}$ hydrogen trajectory.

The isotope effect on the rotational tunnel splitting (ground to first excited state) for a hindered rotor is much larger than that for the free rotor (deuterium energies lowered by a factor of 2), typically lower by a factor of 4-8 in deuterium than in hydrogen in in our calculations. For the IR experiment the first-to-second excited state transition energy (ortho-ortho states, accessible to IR scattering), is lowered in deuterium by much less than a factor of $2 \ldots$ only a few percent for the rotational barriers typical of our simulation.

While the above argument for the IR sidebands containing a $\mathrm{CM}$ and not a rotational mode, is compelling, it raises an ambiguity concerning the assignment of the line at 90 $100 \mathrm{~cm}^{-1}$ as a $1 \rightarrow 2$ rotational transition, if this were to correspond to the line at $75 \mathrm{~cm}^{-1}$ in the deuterium spectrum (i.e., the isotope effect would be around $20 \%$, much more than a few percent).

A possible explanation for these ambiguities lies in our neglect of zero-point energy effects, which may affect the distribution of potential energy wells experienced by the two isotopic species. Since the mass of the guest does not enter into the partition function in our model, we suggest that the identity of the two lines in this satellite must be left open for a future study, in which realistic center-of-mass dynamics of $\mathrm{H}_{2}$ and $\mathrm{D}_{2}$ is incorporated.

Another type of feature which one observes in some IR data is an ortho-para frequency splitting in the main $\nu(\mathrm{HH})$ vibrational band or a sideband. It has been shown to be approximately $5 \mathrm{~cm}^{-1}$ in $\mathrm{Na}-\mathrm{A}$, and $4.5 \mathrm{~cm}^{-1}$ in $\mathrm{Na}-\mathrm{Ca}-\mathrm{A},{ }^{9,55}$ as opposed to $6 \mathrm{~cm}^{-1}$ in the gas phase. This small effect has been calculated by Forster and Frede ${ }^{55}$ and Larin and Cohen de Lara; ${ }^{38}$ and it may be checked for our model as well. We seek

$$
\begin{aligned}
\Delta E= & \left(E_{0}(\nu=1)-E_{0}(\nu=0)\right) \\
& -\left(E_{1}(\nu=1)-E_{1}(\nu=0)\right),
\end{aligned}
$$

where $E_{i}(\nu)$ is the energy of the $i$ th rotational state with vibrational quantum number $\nu$. Anharmonicity in the intramolecular vibration makes $\Delta E$ nonzero; the bond length $d_{\nu}$ increases with $\nu$, and thus the rotational constant, $B_{\nu}$, decreases. Using the formula for $\mathrm{H}_{2},{ }^{57}$

$$
B_{\nu}=60.8 \mathrm{~cm}^{-2}-3.0\left(\nu+\frac{1}{2}\right) \mathrm{cm}^{-1}
$$

allows us to calculate the terms of Eq. (16) ${ }^{58}$ First, we try the model of a purely out-of-plane hindered rotor (presumed relevant to adsorption in $\mathrm{Na}-\mathrm{Ca}-\mathrm{A})^{36}$ with $\lambda=570 \mathrm{~cm}^{-1}$, typical of our system, and find $\Delta E=4.6 \mathrm{~cm}^{-1}$. In the limit of extremely strong hindering of this type, $\mathrm{H}_{2}$ becomes a "planar rotor," and Eq. (17) would give $\Delta E=3 \mathrm{~cm}^{-1}$. In-plane hindering is expected to reduce $\Delta E$ further, for a given $\lambda$.

The experimentally observed ortho-para vibrational splitting is reported to be greater for $\mathrm{Na}-\mathrm{A}$ than $\mathrm{Ca}-\mathrm{Na}-\mathrm{A}$ by $0.5 \mathrm{~cm}^{-1}$; this is a small difference whose significance is difficult to assess at this time. Analysis based on neutron 
experiment assigns roughly the same simple twofold rotational barrier to $\mathrm{H}_{2}$ in $\mathrm{Na}-\mathrm{A}$ as it does to $\mathrm{Ca}-\mathrm{Na}-\mathrm{A}$ : the 6.4 $\mathrm{kJ} / \mathrm{mol}$ mentioned earlier. ${ }^{30}$

We have estimated $\Delta E$ from a sample trajectory in our zeolite model. A trajectory of 500 steps was generated at a temperature of $T=77 \mathrm{~K}$ with the bond length corresponding to $B_{0}$ in Eq. (17), $d_{0}=1.420$ a.u. The same trajectory was then used in an energy calculation assuming a bond length corresponding to $B_{1}, d_{1}=1.457$ a.u. The resulting average value is $\Delta E=4.0 \mathrm{~cm}^{-1}$, with a standard standard deviation of 0.7 , showing that $\Delta E$ varies rather widely with position in the zeolite host. It is relevant to note that our calculation utilizes fixed molecular properties, like polarizability and quadrupole moment (that is to say, our guests are assumed to always be in the ground vibrational state). But variations of these properties with vibrational state do occur. Knowing how they vary is, for example, essential to the calculations of the crystal field-induced shift of IR vibrational bands. The work of Larin and Cohen de Lara ${ }^{37,38}$ indicates that molecular quantities which go into this calculation, like polarizability and quadrupole moment, and hence rotational barriers, would be increased for the first excited vibrational state over the ground state. It has also been found ${ }^{39}$ that changes in these molecular quantities are virtually the same in the gaseous and the absorbed state of $\mathrm{H}_{2}$ in $\mathrm{Na}-\mathrm{A}$; this calculation is supported by observations concerning the anharmonicityinduced IR overtone positions for $\mathrm{H}_{2}$ in A-type zeolites. ${ }^{59}$ Use of the correct first-vibrational-state polarizability and quadrupole moment ${ }^{60}$ in our model was found to increase our calculated $\Delta E$, so that a 500 step trajectory gave $\Delta E$ $=5.5 \mathrm{~cm}^{-1}$, with a standard deviation of 0.9 . It is hard to attach significance to such small disagreements between calculation and experiment; for example, $\Delta E$ was measured to be $7 \mathrm{~cm}^{-1}$ in a recent experiment. ${ }^{24}$ Similarly, good agreement with experiment is reported based on a calculation of Ref. 38. There, the ionicity of the model was $q_{\mathrm{Na}}=0.7 e$; while we use $q_{\mathrm{Na}}=0.6 e$ in the current study.

\section{CONCLUSIONS}

Our combined simulation/experimental study of the adsorption of $\mathrm{H}_{2}$ in $\mathrm{Na}-\mathrm{A}$ clearly demonstrates the remarkable sensitivity of the rotational tunnel splitting to details of the molecular adsorption. Analysis of the experiment was guided by careful simulation studies. The results of simulations include calculations of typical rotational potential energy surfaces, and the corresponding rotational energy levels, with their associated parities. These data suggest that a traditional, simple parametrization of the rotational potential energy be slightly revised to the form of Eq. (15). We have estimated the extent to which the rotational wave function is delocalized in typical adsorption locations, and also within the octagonal doorway of zeolite $\mathrm{Na}-\mathrm{A}$. We have calculated neutron spectra, and noted agreement with experiment (a tunnelsplitting energy in the neighborhood of $30 \mathrm{~cm}^{-1}$ ) as well as disagreement (an absence of the experimentally-seen peak at $\left.70 \mathrm{~cm}^{-1}\right)$.

A combined approach of INS and IR spectroscopies is highly desirable and would no doubt provide the most complete picture of the dynamics of the adsorbate molecule.
There are a number of important extension of this work that would be of interest, including zeolites with divalent cations and those with Bronsted acid sites (H-Y09 or H-ZSM-5), i.e., with significant catalytic activity.

Ideally, as has happened successfully with IR spectroscopy of guests in zeolites, simulation should guide experiment, and experiment should, in turn, correct the simulation model. Ultimately, one may converge on an accurate interaction potential and a suitable mode of simulation of a guest like hydrogen within a zeolite host. Based on this study and the observation of a second peak in the experimental INS spectrum from a possible center-of-mass mode, along with other INS and IR data in the literature, we suggest that a modification of simulations of this type to include center-ofmass dynamics is the next important step. An application that would benefit from such a model is that of gas separation; for example, a study of the separation factor for hydrogen and deuterium within a zeolite host.

\section{ACKNOWLEDGMENTS}

We are grateful to many individuals for their advice during the course of this study; among them E. Cohen de Lara, P. Collings, J. E. Fischer, A. B. Harris, Xiao Li, J. Nicol, P. Papanek, R. Pasternack, T. Stephenson, and D. White. We are very grateful for the technical contributions of J. Boccio, G. Martyna, and T. Reese. We would also like to acknowledge useful contributions by the following undergraduate students at Swarthmore College: C. Anderson and B. Yanoff. The authors would like to thank the Institute Laue-Langevin for the use of their neutron scattering facilities. This research was initiated under N.S.F. V.P.W. Program No. 9103932, and additionally supported by Cottrell College Science Award of Research Corporation No. C-3617, and the Faculty Research Support Fund of Swarthmore College. D.F.C. acknowledges NSF support from Grant No. CHE-9521793. We thank (the former) N.C.S.A. at University of Illinois U.C. as well as the Pittsburgh Supercomputing Center, for grants of Supercomputer time. Work at Los Alamos National Laboratory was supported under the LDRD program. LANL is operated by the University of California under Contract No. W-7045-ENG-36 with the U.S. Department of Energy.

${ }^{1}$ C. R. Anderson, D. F. Coker, J. Eckert, and A. L. R. Bug, J. Chem. Phys. 111, 7599 (1999).

${ }^{2}$ S. Furuyama and H. Inoue, J. Phys. Chem. 87, 1529 (1983).

${ }^{3}$ D. A. Dixon, J. L. Gole, and A. Komornicki, J. Phys. Chem. 92, 1378 (1988).

${ }^{4}$ C. W. Bauschlicher, H. Partridge, and S. R. Langhoff, J. Phys. Chem. 96, 2475 (1992).

${ }^{5}$ L. Koubi, M. Blain, E. Cohen de Lara, and J-M. Leclerq, Chem. Phys. Lett. 217, 544 (1994).

${ }^{6}$ E. R. Cohen de Lara, Mol. Phys. 66, 479 (1989).

${ }^{7}$ J. Sauer, P. Ugliengo, E. Garrone, and V. R. Saunders, Chem. Rev. 94, 2095 (1994).

${ }^{8}$ T. Yamazaki, T. Watanuki, S. Ozawa, and Y. Ogino, Mol. Phys. 73, 649 (1991).

${ }^{9}$ H. Forster, W. Frede, and G. Peters, in Zeolites as Catalysts, Sorbents and Detergent Builders, edited by H. G. Karge and J. Weitkamp (Elseveier Science, Amsterdam, 1989), p. 545.

${ }^{10}$ O. Zakharieva-Pencheva, H. Bose, H. Forster, W. Frede, and M. Grodzicki, J. Mol. Struct. 122, 101 (1985); A. Zecchina and C. Otero Arean, Chem. Soc. Rev. 25, 187 (1996).

${ }^{11}$ A. Sasse and H. Forster, J. Mol. Struct. 349, 97 (1995). 
${ }^{12}$ V. B. Kazansky, V. Yu. Borovkov, and H. G. Karge, J. Chem. Soc., Faraday Trans. 93, 1843 (1997).

${ }^{13}$ R. Kahn, E. Cohen De Lara, and E. Viennet, J. Chem. Phys. 91, 5097 (1989); E. Cohen De Lara and R. Kahn, in Guidelines for Mastering the Properties of Molecular Sieves, edited by D. Barthomeuf et al. (Plenum Press, New York, 1990), p. 169.

${ }^{14}$ T. B. Reed and D. W. Breck, J. Am. Chem. Soc. 78, 5972 (1956); R. W. G. Wyckoff, Crystal Structures, 2nd ed. (Wiley Interscience, New York, 1968), Vol. 4; V. Subramanian and K. Seff, J. Phys. Chem. 81, 2249 (1977); J. J. Pluth and J. V. Smith, J. Am. Chem. Soc. 102, 4704 (1980).

${ }^{15}$ F. Darkrim, A. Aoufi, P. Malbrunot, and D. Levesque, J. Chem. Phys. 112, 5991 (2000)

${ }^{16}$ F. Webster, P. J. Rossky, and R. A. Friesner, Comput. Phys. Commun. 63, 494 (1991); J. K. Cullum and R. A. Willoughby, Lanczos Algorithms for Large, Symmetric Eigenvalue Computations (Birkhauser, Boston, 1985).

${ }^{17}$ M. H. Kalos and P. A. Whitlock, Monte Carlo Methods (Wiley, New York, 1986)

${ }^{18}$ D. A. McQuarrie, Statistical Mechanics (Harper and Row, New York, 1973), pp. 104 and 105.

${ }^{19}$ H. Forster and M. Schuldt, J. Chem. Phys. 66, 5237 (1977).

${ }^{20}$ M. F. Crawford and R. E. MacDonald, Can. J. Phys. 36, 1022 (1958); D. A. Dows and A. D. Buckingham, J. Mol. Spectrosc. 12, 189 (1964).

${ }^{21}$ H. Forster and M. Schuldt, J. Mol. Struct. 47, 339 (1978).

${ }^{22}$ F. Wakabayashi, T. Fujino, J. N. Kondo, K. Domen, and C. Hirose, J. Phys. Chem. 99, 14085 (1995).

${ }^{23}$ K. Beck, H. Pfeifer, and B. Staudte, J. Chem. Soc., Faraday Trans. 89, 3995 (1993)

${ }^{24}$ F. Stephanie-Victoire and E. Cohen de Lara, J. Chem. Phys. 109, 6469 (1998).

${ }^{25}$ S. Bordiga, E. Garrone, C. Lamberti, A. Zecchina, C. O. Arean, V. B. Kazansky, and L. M. Kustov, J. Chem. Soc., Faraday Trans. 90, 3367 (1994).

${ }^{26}$ V. B. Kazansky, V. Yu. Borovkov, and H. G. Karge, Proceedings of the 12th International Zeolite Conference (Materials Research Society, 1999).

${ }^{27}$ L. M. Kustov and V. B. Kazansky, J. Chem. Soc., Faraday Trans. 87, 2675 (1991).

${ }^{28}$ A. Y. Khodakov, L. M. Kustov, V. B. Kazansky, and C. Williams, J. Chem. Soc., Faraday Trans. 88, 3251 (1992).

${ }^{29}$ J. M. Nicol, J. Eckert, and J. Howard, J. Phys. Chem. 92, 7117 (1988).

${ }^{30}$ J. Eckert, J. M. Nicol, J. Howard, and F. R. Trouw, J. Phys. Chem. 100, 10646 (1996).

${ }^{31}$ J. Eckert, F. Trouw, A. L. R. Bug, and R. Lobo, Proceedings of the 12th International Zeolite Conference (Materials Research Society, Pittsburgh, PA, 1999).

${ }^{32}$ H. Jobic, Spectrochim. Acta, Part A 48, 293 (1992).

${ }^{33}$ R. Stockmeyer, Zeolites 5, 393 (1985).

${ }^{34}$ I. J. Braid, J. Howard, J. M. Nicol, and J. Tomkinson, Zeolites 7, 214 (1987).

${ }^{35}$ J. Eckert, F. R. Trouw, and M. McMenomy, in Materials Research Using Cold Neutrons at Pulsed Neutron Sources (New World Scientific Publishing, Singapore, 1998).

${ }^{36}$ A. V. Larin and V. S. Parbuzin, Mol. Phys. 77, 869 (1992).

${ }^{37}$ A. V. Larin and E. Cohen De Lara, J. Chem. Phys. 101, 8130 (1994).

${ }^{38}$ A. V. Larin and E. Cohen De Lara, Mol. Phys. 88, 1399 (1996).

${ }^{39}$ A. V. Larin, F. Jousse, L. Leherte, and D. P. Vercauteren, Chem. Phys. Lett. 274, 345 (1977).

${ }^{40}$ A. L. R. Bug and G. J. Martyna, Chem. Phys. 261, 89 (2000).

${ }^{41}$ M. F. Falcetta, J. L. Pazun, M. J. Dorko, D. Kitchen, and P. E. Siska, J. Phys. Chem. 97, 1011 (1993).

${ }^{42}$ D. M. Bishop and S. M. Cybulski, Chem. Phys. Lett. 230, 177 (1994)

${ }^{43}$ J. P. Bouchaud, E. Cohen De Lara, and R. Kahn, Europhys. Lett. 17, 583 (1992).
${ }^{44}$ D. Basmadjian, Can. J. Chem. 38, 141 (1960).

${ }^{45}$ E. Cohen De Lara (private communication).

${ }^{46}$ D. M. Ruthven, Principles of Adsorption and Adsorption Processes (Wiley, New York, 1984).

${ }^{47}$ L. A. Curtiss and J. A. Pople, J. Phys. Chem. 92, 894 (1988).

${ }^{48}$ It is possible to quantitatively relate the intramolecular vibration of $\mathrm{H}_{2}$ to the strength and direction of the electric field, as, for example, in Refs. 6, 8 , and $24, \mathrm{H}_{2}$. It is quite unlikely that the binding of $\mathrm{H}_{2}$ is directly side-on to a cation, but rather that it is asymmetric; as the authors of Ref. 7 observe, "One possibility for an asymmetric interaction is a dual site adsorption involving a cation and a nearby oxygen atom."

${ }^{49}$ The energy and momentum transferred during neutron scattering are related in a way which must be calculated, given the the detector angle (TOF technique) or on the incident energy (BT-4 technique). Certainly, each rotational line will not draw uniformly from the chosen distribution of wave numbers. While our choice of a range of wave numbers was made in the hope of giving a spectrum with realistic relative peak heights, it was not chosen in collaboration with experimentors, to accurately reproduce the conditions of any particular experiment.

${ }^{50}$ J. Eckert, J. M. Nicol, and T. Vogt (unpublished result).

${ }^{51}$ J. Eckert and J. M. Nicol (unpublished).

${ }^{52}$ E. Cohen de Lara, Phys. Chem. Chem. Phys. 1, 501 (1999).

${ }^{53}$ A. A. Evett, J. Chem. Phys. 33, 789 (1960); T. B. MacRury and J. R. Sams, Mol. Phys. 19, 337 (1970); 20, 57 (1971).

${ }^{54} \mathrm{~A}$ full description of $\mathrm{H}_{2}$ adsorption in $\mathrm{ZnNaA}$ may be slightly more complicated still. One of us (J.E.) has performed INS experiments on both $\mathrm{Zn} 4.2 \mathrm{Na3}$.6-A and Zn3.06Na5.87-A. Both of these samples show a new peak at approximately $0.5 \mathrm{~B}$, which was not resolved in the older data of Braid et al. Reduction of $\mathrm{Zn}$ content reduces the relative size of the peak at $1.2 \mathrm{~B}$, so we might assume that it is associated (at least partially) with $\mathrm{Zn}$ sites while the peak at $0.5 \mathrm{~B}$ is associated (at least partially) with $\mathrm{Na}$ sites. The peak at $1.2 \mathrm{~B}$ should, based on our potential model, correspond to a mixture of $g\langle-\rangle 1$ and $1\langle-\rangle 2$ transitions; while the peak at $2.5 \mathrm{~B}$ corresponds to $g\langle-\rangle 2$; all from the Zn-associated sites. Peaks corresponding to $g\langle-\rangle 2$ and $1\langle-\rangle 2$ from the Na-associated sites would fall at frequencies too high to be observed in the new data.

${ }^{55}$ R. Kahn, E. Cohen De Lara, P. Thorel, and J. L. Ginoux, Zeolites 2, 260 (1982); E. Cohen De Lara and R. Kahn, Proceedings of the 6th International Zeolite Conference (Butterworths, Guildford, 1984). In these references, the binding of methane is considered. For this guest, binding occurs in the direction of $\mathrm{Na}_{\text {II }}$ but in proximity to both $\mathrm{Na}_{\text {II }}$ and $\mathrm{Na}_{\text {III }}$ ions; and the latter is identified as the source of the strongest binding.

${ }^{56}$ H. Bose, H. Forster, and W. Frede, Chem. Phys. Lett. 138, 401 (1987).

${ }^{57}$ D. A. McQuarrie, Quantum Chemistry (University Science Books, Mill Valley, California, 1983), Ch. 10.

${ }^{58}$ An alternative choice of intermolecular distances for these calculations is suggested by the work of Refs. 38 and 39, where a solution to the vibrational Schrödinger equation gives 1.448 a.u. and 1.544 a.u. for the ground and first excited state radii. (The first of these is roughly equal to the bond length chosen for hydrogen for the present study.) These larger values would, within our simple rigid-rotor theory, give a gas phase ortho-para IR vibrational splitting of $13.71 \mathrm{~cm}^{-1}$, which is more than twice the experimental value. While our predictions for the ortho-para splitting could have been renormalized, and given in units of the rotational constant of free hydrogen, we instead chose to work with the parametrization of Eq. (17), which gives an ortho-para splitting of $6 \mathrm{~cm}^{-1}$ for free $\mathrm{H}_{2}$, as one expects.

${ }^{59}$ H. Forster and W. Frede, Infrared Phys. 24, 151 (1984).

${ }^{60} \mathrm{We}$ calculate the fractional increase of quadrupole moment and polarizability from Eq. (17) of Ref. 37, and apply that increase to the corresponding parameters of our model. 